\title{
Direct numerical simulation study of statistically stationary propagation of a reaction wave in homogeneous turbulence
}

\author{
Rixin $\mathrm{Yu}^{1}$ and Andrei N. Lipatnikov ${ }^{2}$ \\ ${ }^{1}$ Division of Fluid Mechanics, Department of Energy Sciences, Lund University, 22100 Lund, Sweden \\ ${ }^{2}$ Department of Mechanics and Maritime Sciences, Chalmers University of Technology, Göteborg, 412 96, Sweden
}

(Received 14 March 2017; revised manuscript received 14 May 2017; published 1 June 2017)

\begin{abstract}
A three-dimensional (3D) direct numerical simulation (DNS) study of the propagation of a reaction wave in forced, constant-density, statistically stationary, homogeneous, isotropic turbulence is performed by solving Navier-Stokes and reaction-diffusion equations at various (from 0.5 to 10 ) ratios of the rms turbulent velocity $U^{\prime}$ to the laminar wave speed, various (from 2.1 to 12.5 ) ratios of an integral length scale of the turbulence to the laminar wave thickness, and two Zeldovich numbers $\mathrm{Ze}=6.0$ and 17.1. Accordingly, the Damköhler and Karlovitz numbers are varied from 0.2 to 25.1 and from 0.4 to 36.2, respectively. Contrary to an earlier DNS study of self-propagation of an infinitely thin front in statistically the same turbulence, the bending of dependencies of the mean wave speed on $U^{\prime}$ is simulated in the case of a nonzero thickness of the local reaction wave. The bending effect is argued to be controlled by inefficiency of the smallest scale turbulent eddies in wrinkling the reaction-zone surface, because such small-scale wrinkles are rapidly smoothed out by molecular transport within the local reaction wave.
\end{abstract}

DOI: 10.1103/PhysRevE.95.063101

\section{INTRODUCTION}

The critical point of the theory of turbulent reacting flows stems from averaging reaction rates subject to fluctuations in the local concentrations and temperature $T$. The problem is particularly severe in the case of premixed burning, because (i) the rates of reactions that control heat release depend on $T$ in a highly nonlinear manner, (ii) the magnitudes of the temperature fluctuations are typically large, and (iii) these fluctuations exhibit a wide range of length and time scales [1-3]. Accordingly, even most advanced models of a highly nonlinear multiscale problem such as the influence of turbulence on premixed combustion invoke a number of simplifications, i.e., highlight certain local effects but ignore many others. Such models are commonly assessed by (i) applying them to Reynolds-averaged Navier-Stokes (RANS) or large eddy simulation (LES) of an experiment and (ii) comparing obtained numerical results with measured data. However, such a test does not offer an opportunity to scrutinize the model foundations, e.g., to reveal a particular assumption that causes eventual disagreement between the numerical and experimental data.

Recent research trends in the field of turbulent burning indicate that a breakthrough in assessing and ranking various models by scrutinizing their foundations becomes possible thanks to the rapid development of computer hardware and software, which has made direct numerical simulation (DNS) of turbulent reacting flows feasible. Progress made in this area over the past years was very impressive. In particular, the leading research groups succeeded already in 3D DNSs of highly turbulent premixed flames by allowing for density variations and complex combustion chemistry [4-10]. Moreover, DNS of laboratory flames were also performed [11,12]. However, while advancement to simulations of increasingly challenging problems was in the focus of the numerical combustion community over the past decade, unique opportunities offered by DNS for scrutinizing foundations of turbulent combustion models did not seem to be exploited in full measure.

The point is that the vast majority of models of the influence of turbulence on premixed combustion (i) deal with single-step chemistry and (ii) neglect density variations, with a few exceptions [13-16]. Accordingly, to explore foundations of such models, heavy DNS with complex chemistry are not necessary, but extension of the range of studied conditions appears to be of paramount importance. Indeed, several recent DNS studies of variable-density single-step-chemistry turbulent premixed flames [17-22] aimed at assessing models of premixed turbulent burning and exploring physical mechanisms of turbulent combustion. However, to perform a target-directed investigation of many models, DNS of a constant-density reacting flow makes sense, because problems addressed by the models and simulations are as close as possible in such a case. Nevertheless, DNS of a constantdensity turbulent reacting flow was beyond the focus of the combustion community in this century.

The goal of the present work is to fill this gap by highlighting and discussing certain effects, which are observed in constant-density DNS, but appear to be of importance for understanding and modeling premixed turbulent flames. In the first two papers of the present series [23,24], we explored such effects by analyzing DNS data obtained in computations of self-propagation of an infinitely thin interface in homogeneous isotropic turbulence. The present work extends the previous study by (i) considering a reaction wave of a nonzero thickness, with all other things being equal, and (ii) investigating the influence of the thickness on the mean wave characteristics such as its speed and thickness, turbulent scalar transport within the mean wave, etc. Such an extension of the previous work appears to be a reasonable second step in a step-by-step study of a role played by various phenomena (self-propagation of an infinitely thin front, nonzero thickness of a reaction wave, Lewis number and preferential diffusion effects, density 
variations, and, finally, complex chemistry) in premixed turbulent combustion. ${ }^{1}$

The present paper is organized as follows. In the next two sections, governing equations are briefly summarized and DNS attributes are reported. The obtained results are discussed in Sec. IV, followed by our conclusions.

In order to stress the difference between constant-density turbulent flow and turbulent burning accompanied by significant density variations, we will avoid terms flame and flamelet when discussing constant-density DNS data in the rest of the paper. For instance, a statistically planar spatial region that envelops all local reaction waves at a single instant will be called a mean wave or mean wave brush. The mean speed of this mean wave brush with respect to the mean flow will be called a mean wave speed. Nevertheless, because the present study aims at clarifying basic issues associated with premixed turbulent combustion, the same symbol $\delta_{T}$ will designate both a mean flame brush thickness and a mean wave thickness obtained in the present DNS. Similarly, the same symbol $S_{T}$ will designate both a turbulent flame speed or burning velocity and a mean wave speed obtained in the present DNS. Furthermore, the speed of the self-propagation of the wave will be designated with symbol $S_{L}$, which is associated with the laminar flame speed in the combustion literature. Finally, we will use words combustion and flame when comparing the present DNS results with various models of premixed turbulent burning or experimental and DNS data obtained from flames.

\section{GOVERNING EQUATIONS}

The background turbulent flow is described by the constantdensity continuity

$$
\nabla \cdot u=0
$$

and Navier-Stokes

$$
\frac{\partial \boldsymbol{u}}{\partial t}+(\boldsymbol{u} \cdot \nabla) \boldsymbol{u}=-\rho^{-1} \nabla p+v \nabla^{2} \boldsymbol{u}+\boldsymbol{f}
$$

equations, where $t$ is time; $\boldsymbol{u}$ is the flow velocity vector; $\rho$, $v$, and $p$ are the density, kinematic viscosity, and pressure, respectively; and a vector function $f$ is added in order to maintain constant turbulence intensity by using energy forcing at low wave numbers.

Propagation of a reaction wave of a nonzero thickness is modeled by the following convection-diffusion-reaction equation:

$$
\frac{\partial c}{\partial t}+\boldsymbol{u} \cdot \nabla c=\mathcal{D} \nabla^{2} c+W
$$

\footnotetext{
${ }^{1}$ As kindly noted by a reviewer, the first three steps were already passed for a simpler relevant problem, i.e., propagation of a hydrodynamically passive reaction wave through a stationary twodimensional (2D) array of single-scale vortices [25-28]. However, Kagan and Sivashinsky [26] noted that certain results computed by them might not "hold for turbulent combustion" and suggested to extend their "study over multiscale, time-dependent and random velocity fields" (p. 229). The present work responds to such a request in the 3D case.
}

for a scalar field $c$, which is equal to zero and unity in fresh reactants and products, respectively. The molecular diffusivity $\mathcal{D}$ is set to be constant and the reaction rate

$$
W=\frac{1}{1+\tau} \frac{1-c}{\tau_{R}} \exp \left[-\frac{\operatorname{Ze}(1+\tau)^{2}}{\tau(1+\tau c)}\right]
$$

depends on $c$ in a highly nonlinear manner. Here, $\tau_{R}$ is a constant reaction time scale, $\tau=6$, and the parameter Ze, called the Zeldovich number via an analogy with premixed combustion, is specified in Tables II and III in Sec. III. To further draw the analogy with combustion, (i) a sum $(1+$ $\tau)$ is counterpart of the density ratio $\rho_{u} / \rho_{b}$ in a premixed flame, where subscripts $u$ and $b$ designate unburned and burned gas, respectively, and (ii) the scalar $c$ is associated with the combustion progress variable, which is equal to $1-y / y_{u}=$ $\left(T-T_{u}\right) /\left(T_{b}-T_{u}\right)$ if the Lewis number Le $=a / \mathcal{D}=1$. Here, $y$ is the mass fraction of the deficient reactant, and $a$ is the molecular heat diffusivity of the mixture.

In the present work, DNS cases were set up by specifying (i) Ze, (ii) the speed $S_{L}$ of the steady propagation of the planar reaction wave in quiescent flow, and (iii) the wave thickness $\delta_{F}=\mathcal{D} / S_{L}$. To do so, the diffusivity $\mathcal{D}$ and time scale $\tau_{R}$ were tuned in simulations of planar one-dimensional (1D) laminar waves in order to obtain required $S_{L}$ and $\delta_{F}$.

Results obtained in the present DNS will be compared with recent DNS data [24] computed by solving the level-set equation [29]

$$
\frac{\partial G}{\partial t}+\boldsymbol{u} \cdot \nabla G=S_{L}|\nabla G|
$$

instead of Eq. (3), with all other things being equal. Here, $G$ is a signed distance function to the closest interface associated with $G(\boldsymbol{x}, t)=0$, the field of $c(\boldsymbol{x}, t)=H[G(\boldsymbol{x}, t)]$ is the counterpart of the $c$ field modeled using Eq. (3) in the present work, and $H(z)$ is the Heaviside function. The interface speed $S_{L}$ was kept constant by Yu et al. [24].

\section{NUMERICAL METHOD AND SIMULATION CONDITIONS}

The computational domain is a fully periodic rectangular box of size of $L_{x} \times L_{y} \times L_{z}$ with $L_{x}=4 L_{y}=4 L_{z}=4 L$. The domain is discretized using a uniform staggered Cartesian grid of $N_{x} \times N_{y} \times N_{z}$ cells with $N_{x}=4 N_{y}=4 N_{z}$. Most results reported in the present paper were obtained using three different domains (three different $L$ ), but retaining the same spatial resolution $\Delta x=\Delta y=\Delta z=L_{x} / N_{x}=$ $L_{y} / N_{y}=L_{z} / N_{z}$. In all cases, the viscosity $v$ is kept constant and equal to $1.56 \times 10^{-4} \mathrm{~m}^{2} / \mathrm{s}$.

Simulations were performed using a simplified in-house DNS solver [30] developed for low-Mach-number reacting flows and equipped with a standalone stiff chemistry solver for a general kinetic mechanism. The solver was already applied to various reacting flow systems [31-34]. In the original solver, the temporal integration of the governing equations is based on a second-order symmetrical Strang splitting algorithm. In the present work, the solver is simplified because the source term $W(c)$ given by Eq. (4) is not stiff. In particular, when numerically integrating Eq. (3), temporal advancement is performed for a full time-step $\left(\Delta t=t^{n+1}-t^{n}=0.029 \Delta x / U^{\prime}\right)$ 
using the Adams-Bashforth method in multiple subtime steps, i.e., $\Delta t_{*}=\Delta t / K=t_{*}^{k+1}-t_{*}^{k}, k=0, \ldots, K, t_{*}^{0}=t^{n}, K>1$. Accordingly,

$$
\begin{aligned}
\frac{c\left(t_{*}^{k+1}\right)-c\left(t_{*}^{k}\right)}{\Delta t_{*}}= & \frac{3}{2} \mathbb{D}\left(t_{*}^{k}\right)-\frac{1}{2} \mathbb{D}\left(t_{*}^{k-1}\right)+\left(1+a_{k}\right) \mathbb{H}\left(t^{n}\right) \\
& -a_{k} \mathbb{H}\left(t^{n-1}\right)
\end{aligned}
$$

where $a_{k}=(k+1 / 2) / K$ is an interpolation coefficient and $\mathbb{D}=\mathcal{D} \nabla^{2} c$ and $\mathbb{H}=-\boldsymbol{u} \cdot \nabla c+W$ are the diffusion term and the sum of the convection and reaction terms, respectively. In order to reduce the computational costs, the $\mathbb{H}$ term is evaluated for the full time step. Such a solution is feasible, because the stability of the numerical scheme is mainly controlled by the diffusion term.

The convection term is discretized using a fifth-order weighted essentially nonoscillatory (WENO) scheme [35], whereas all other spatial terms are discretized using sixth-order center schemes.

The constant-density flow solver is largely identical to the solver used by us earlier [23,24], but the multigrid solver [36] for the constant-coefficient Poisson equation with periodic boundaries is replaced with an accurate spectrum solver using an open-source, parallel version of FFTW3 (mpi-fftw). The DNS code is implemented in a vector form enabling 1D, 2D, and 3D simulations.

The initial turbulence field is generated by synthesizing prescribed Fourier waves [37] with an initial rms velocity $U_{0}^{\prime}$ and a turbulence length scale $\Lambda_{0}=L / 4$. As a special case by Lamorgese et al. [38], the forcing function $\boldsymbol{f}(\boldsymbol{x}, t)=$ $\sum_{\kappa} \hat{\boldsymbol{f}}_{\boldsymbol{\kappa}}(t) \exp (\boldsymbol{i} \boldsymbol{\kappa} \cdot \boldsymbol{x})$ is invoked in order to maintain statically stationary turbulence. Here,

$$
\hat{\boldsymbol{f}}_{\boldsymbol{\kappa}}(t)=\frac{\langle\varepsilon\rangle 1_{\boldsymbol{\kappa}-\boldsymbol{\kappa}_{r}(t)}}{\hat{\boldsymbol{u}}_{\boldsymbol{\kappa}}(t) \cdot \hat{\boldsymbol{u}}_{\boldsymbol{\kappa}}^{*}(t)} \hat{\boldsymbol{u}}_{\boldsymbol{\kappa}}(t)
$$

is the Fourier mode of $\boldsymbol{f}$ in the wave-number $\boldsymbol{\kappa}$ space,

$$
\varepsilon=2 v S_{i j} S_{i j}=\frac{v}{2}\left(\frac{\partial u_{i}}{\partial x_{j}}+\frac{\partial u_{j}}{\partial x_{i}}\right)\left(\frac{\partial u_{i}}{\partial x_{j}}+\frac{\partial u_{j}}{\partial x_{i}}\right)
$$

is the dissipation rate, and the bracket $\langle\cdot\rangle$ designates averaging over entire domain. The caret operator designates the complex Fourier mode $\hat{q}_{\boldsymbol{\kappa}}(t)=\langle q(\boldsymbol{x}, t) \exp (-i \boldsymbol{\kappa} \cdot \boldsymbol{x})\rangle$ for any $q$, a superscript star denotes a complex conjugate, $1_{\kappa-\kappa_{r}}=1$ when $\boldsymbol{\kappa}=\boldsymbol{\kappa}_{r}$ or vanishes otherwise, $\boldsymbol{\kappa}_{r}=$ $\left\{2 \pi m_{x} / L_{x}, 2 \pi m_{y} / L_{y}, 2 \pi m_{z} / L_{z}\right\}$ is a randomly selected (at each time step) nonzero wave-number vector within a userspecified lower wave-number band, i.e., $\left|\boldsymbol{\kappa}_{r}\right| \leqslant \kappa_{f}$, and $\boldsymbol{m}$ is a random integer vector. By adopting the same forcing technique with $\kappa_{f} / \kappa_{0}=3$, where $\kappa_{0}=2 \pi / L$, Yu et al. [23,24] showed that (i) the rms velocity $U^{\prime}$ was maintained as the initial value, i.e., $U^{\prime}=U_{0}^{\prime}$, (ii) the normalized averaged dissipation rate $\left(\Lambda_{0} / U_{0}^{\prime 3}\right)\langle\varepsilon\rangle$ fluctuated slightly above $3 / 2$ after a short period $\left(t<\tau_{t}^{0}=\Lambda_{0} / U_{0}^{\prime}\right)$ of rapid transition from the initial artificially synthesized flow to developed turbulence, (iii) the forced turbulence achieved statistical homogeneity and isotropy over the entire domain (see also Fig. 1), and (iv) the energy spectrum showed a sufficiently wide range of the Kolmogorov scaling $(-5 / 3)$ at $\operatorname{Re}_{0}=U_{0}^{\prime} \Lambda_{0} / v=200$.

Most present results were obtained at the same ratio of $\kappa_{f} / \kappa_{0}=3$. In such a case, three basic turbulence fields were

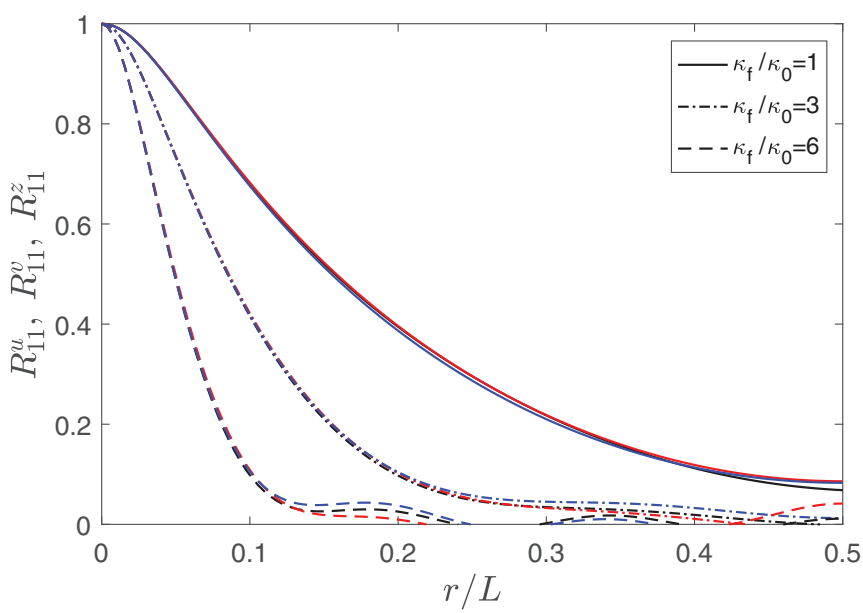

FIG. 1. Longitudinal autocorrelation functions $R_{11}^{u}$ (black), $R_{11}^{v}$ (red), and $R_{11}^{w}$ (blue) obtained from statistically stationary turbulence maintained using three forcing radii; $\kappa_{f} / \kappa_{0}=1$ (solid lines), 3 (dashed-dotted lines), and 6 (dashed lines). $\mathrm{Re}_{0}=200$.

generated by specifying three different values $\mathrm{Re}_{0}=50,100$, or 200 of the initial turbulent Reynolds number, which was increased by increasing the domain size $L$. The first three lines in Table I show characteristics of these three fields, calculated after the forced turbulence reached statistical stationarity, i.e., at $t>5 \tau_{t}^{0}$. Here, $L_{11}=\int_{0}^{L / 2} R_{11}^{u}(r) d r \approx$ $\int_{0}^{L / 2} R_{11}^{v}(r) d r \approx \int_{0}^{L / 2} R_{11}^{w}(r) d r$ and $\tau_{t}=L_{11} / U^{\prime}$ are the longitudinal integral length scale and the corresponding time scale, respectively, $\eta=\left(v^{3} / \overline{\langle\varepsilon\rangle}\right)^{1 / 4}$ and $\tau_{\eta}=(\nu / \overline{\langle\varepsilon\rangle})^{1 / 2}$ are the Kolmogorov length and time scales, respectively, $\overline{\langle\varepsilon\rangle}$ is the dissipation rate averaged both over the computational domain and time, $\Lambda=\left(3 U^{\prime 2} / 2\right)^{3 / 2} / \overline{\langle\varepsilon\rangle}$ is another turbulence length scale, which is often used in DNS papers, and $\operatorname{Re}_{t}=U^{\prime} L_{11} / v$ and $\operatorname{Re}_{\Lambda}=U^{\prime} \Lambda / v$ are the Reynolds numbers based on the length scales $L_{11}$ and $\Lambda$, respectively. The autocorrelation functions $\quad R_{11}^{u}(r)=\overline{\langle u(x, y, z) u(x+r, y, z)\rangle} / U^{\prime 2}$, $R_{11}^{v}(r)=\overline{\langle v(x, y, z) v(x, y+r, z)\rangle} / U^{\prime 2}, \quad$ and $\quad R_{11}^{w}(r)=$ $\overline{\langle w(x, y, z) v(x, y, z+r)\rangle} / U^{\prime 2}$, averaged over the transverse coordinates and time, are shown in dot-dashed lines in Fig. 1, which indicates the isotropy of the generated turbulence.

Because a reaction wave does not affect turbulence in the case of constant $\rho$ and $\nu$, the flow statistics were the same in all cases that had different $S_{L}$, but the same $\operatorname{Re}_{0}$ and the same $\kappa_{f} / \kappa_{0}$. Statistically, the same turbulence fields were used by Yu et al. $[23,24]$ in the previous DNS study of self-propagation of an infinitely thin interface.

TABLE I. Mean flow characteristics of forced maintained turbulence.

\begin{tabular}{lcccccccccc}
\hline \hline $\operatorname{Re}_{0}$ & $N_{x} \times N_{y} \times N_{z}$ & $\frac{\kappa_{f}}{\kappa_{0}}$ & $\frac{\Lambda}{L_{y}}$ & $\operatorname{Re}_{\Lambda}$ & $\frac{L_{11}}{L_{y}}$ & $\operatorname{Re}_{t}$ & $\frac{\eta}{\Delta x}$ & $\frac{\tau_{t}}{\tau_{t}^{0}}$ & $\frac{\tau_{t}}{\tau_{\eta}}$ & $\frac{L_{11}}{\eta}$ \\
\hline 50 & $256 \times 64^{2}$ & 3 & 0.20 & 41 & 0.13 & 26 & 0.68 & 0.51 & 5.5 & 12 \\
100 & $512 \times 128^{2}$ & 3 & 0.26 & 105 & 0.12 & 48 & 0.87 & 0.47 & 6.2 & 17 \\
200 & $1024 \times 256^{2}$ & 3 & 0.30 & 241 & 0.11 & 86 & 1.07 & 0.42 & 7.4 & 25 \\
200 & $1024 \times 256^{2}$ & 1 & 0.71 & 566 & 0.20 & 158 & 1.32 & 0.79 & 9.1 & 38 \\
200 & $1024 \times 256^{2}$ & 6 & 0.14 & 114 & 0.06 & 50 & 0.88 & 0.25 & 6.4 & 18 \\
$50^{*}$ & $1024 \times 256^{2}$ & 3 & 0.20 & 41 & 0.13 & 26 & 2.69 & 0.51 & 5.5 & 12 \\
\hline \hline
\end{tabular}


TABLE II. Basic DNS cases.

\begin{tabular}{|c|c|c|c|c|c|c|c|c|c|c|c|}
\hline Case & $\operatorname{Re}_{0}$ & $\kappa_{f} / \kappa_{0}$ & $L_{11} / L_{y}$ & $\mathrm{Ze}$ & $U^{\prime} / S_{L}$ & $L_{11} / \delta_{F}$ & $\mathrm{Da}$ & $\mathrm{Ka}$ & $\delta_{F} / \eta$ & $\bar{\delta}_{T} / L_{11}$ & $\bar{S}_{T} / U^{\prime}$ \\
\hline $05-21$ & 50 & 3 & 0.13 & 6.0 & 0.5 & 2.1 & 4.1 & 1.3 & 5.9 & 1.43 & 2.18 \\
\hline $1-21$ & 50 & 3 & 0.13 & 6.0 & 1.0 & 2.1 & 2.1 & 2.7 & 5.9 & 2.05 & 1.31 \\
\hline $2-21$ & 50 & 3 & 0.13 & 6.0 & 2.0 & 2.1 & 1.0 & 5.4 & 5.9 & 2.86 & 0.90 \\
\hline $5-21$ & 50 & 3 & 0.13 & 6.0 & 5.0 & 2.1 & 0.4 & 13.4 & 5.9 & 3.99 & 0.57 \\
\hline $10-21$ & 50 & 3 & 0.13 & 6.0 & 10.0 & 2.1 & 0.2 & 26.9 & 5.9 & 5.22 & 0.40 \\
\hline $05-37$ & 100 & 3 & 0.12 & 6.0 & 0.5 & 3.7 & 7.5 & 0.8 & 4.6 & 1.57 & 2.45 \\
\hline $1-37$ & 100 & 3 & 0.12 & 6.0 & 1.0 & 3.7 & 3.7 & 1.7 & 4.6 & 2.43 & 1.69 \\
\hline $2-37$ & 100 & 3 & 0.12 & 6.0 & 2.0 & 3.7 & 1.9 & 3.3 & 4.6 & 3.22 & 1.27 \\
\hline $5-37$ & 100 & 3 & 0.12 & 6.0 & 5.0 & 3.7 & 0.7 & 8.4 & 4.6 & 4.28 & 0.80 \\
\hline $10-37$ & 100 & 3 & 0.12 & 6.0 & 10.0 & 3.7 & 0.4 & 16.7 & 4.6 & 4.99 & 0.55 \\
\hline $05-67$ & 200 & 3 & 0.11 & 6.0 & 0.5 & 6.7 & 13.5 & 0.6 & 3.8 & 1.78 & 2.77 \\
\hline $1-67$ & 200 & 3 & 0.11 & 6.0 & 1.0 & 6.7 & 6.7 & 1.1 & 3.8 & 2.69 & 2.06 \\
\hline $2-67$ & 200 & 3 & 0.11 & 6.0 & 2.0 & 6.7 & 3.4 & 2.2 & 3.8 & 3.43 & 1.61 \\
\hline $5-67$ & 200 & 3 & 0.11 & 6.0 & 5.0 & 6.7 & 1.3 & 5.5 & 3.8 & 4.34 & 1.11 \\
\hline $10-67$ & 200 & 3 & 0.11 & 6.0 & 10.0 & 6.7 & 0.7 & 11.0 & 3.8 & 4.74 & 0.76 \\
\hline $05-125$ & 200 & 1 & 0.20 & 6.0 & 0.5 & 12.5 & 25.1 & 0.4 & 3.0 & 1.47 & 3.00 \\
\hline $1-125$ & 200 & 1 & 0.20 & 6.0 & 1.0 & 12.5 & 12.5 & 0.7 & 3.0 & 2.15 & 2.23 \\
\hline $2-125$ & 200 & 1 & 0.20 & 6.0 & 2.0 & 12.5 & 6.3 & 1.4 & 3.0 & 2.77 & 1.80 \\
\hline $5-125$ & 200 & 1 & 0.20 & 6.0 & 5.0 & 12.5 & 2.5 & 3.6 & 3.0 & 3.69 & 1.42 \\
\hline $10-125$ & 200 & 1 & 0.20 & 6.0 & 10.0 & 12.5 & 1.3 & 7.2 & 3.0 & 4.27 & 1.00 \\
\hline
\end{tabular}

In the present work, two more turbulence fields were generated by setting $\kappa_{f} / \kappa_{0}$ equal to 1 or 6 , see the fourth and fifth lines in Table I, in order to change ratios of $L_{11} / L$ and $\Lambda / L$ by retaining the same $U^{\prime}$ and $v$ [39]. As shown in Fig. 1, a smaller forcing radius yields wider autocorrelation functions and, therefore, larger $L_{11}$.

It is worth stressing that the fields obtained at (i) $\mathrm{Re}_{0}=$ 100 and $\kappa_{f} / \kappa_{0}=3$ and (ii) $\operatorname{Re}_{0}=200$ and $\kappa_{f} / \kappa_{0}=6$ are characterized by almost equal $\operatorname{Re}_{t}$ or $L_{11}$, but a ratio of $L_{11} / L$ is larger by a factor of about two in the former case. Accordingly, these two turbulence fields were used in order to gain insight into eventual influence of the relative width $L / L_{11}$ of the computational domain on obtained results, with all other things being approximately equal. As will be shown later, such an influence is weak, because the autocorrelation functions vanish (if $\kappa_{f} / \kappa_{0} \geqslant 3$ ) at distance $r$ equal to half the width of the computational domain; see Fig. 1.

In the five aforementioned cases, the Kolmogorov length scale $\eta$ was on the order of the grid cell size $\Delta x$, thus implying sufficient grid resolution. Nevertheless, in order to assess sensitivity of computed results to numerical resolution, highly resolved simulations, see the bottom line in Table I, were also run.

Table II reports characteristics ${ }^{2}$ of 20 basic DNS cases studied in the present work. There are four sets of five cases each, with the first, second, third, and fourth sets being associated with the four turbulence fields addressed in the four first lines in Table I, respectively. The first three sets are characterized by the same $\kappa_{f} / \kappa_{0}=3$ and $L_{11} / L \approx 0.1$, whereas the fourth set is characterized by $\kappa_{f} / \kappa_{0}=1$ and $L_{11} / L \approx 0.06$. Moreover, all 20 cases are characterized by the same Zeldovich number $\mathrm{Ze}$ and the same laminar wave

\footnotetext{
${ }^{2}$ The fully developed mean wave thickness $\bar{\delta}_{T}$ and speed $\bar{S}_{T}$, reported in the last two columns, will be discussed in Sec. IV.
}

thickness $\delta_{F}$. These cases cover four different values of $\operatorname{Re}_{t}$, four different values of $L_{11} / \delta_{F}$, and five different values of $U^{\prime} / S_{L}$, which was changed by varying $S_{L}$, i.e., $\tau_{R}$ and $\mathcal{D}$ in Eq. (4). Each basic case is named using two numbers, a ratio of $U^{\prime} / S_{L}$ and a ratio of $L_{11} / \delta_{F}$. For instance, the name 05-21 or 10-125 of the first or last case, respectively, means that $U^{\prime} / S_{L}=0.5$ or 10 , respectively and $L_{11} / \delta_{F}=2.1$ or 12.5 , respectively.

Within each set, all five cases are characterized by the same $\operatorname{Re}_{t}$, the same $L_{11} / \delta_{F}$, but $U^{\prime} / S_{L}$ and the Karlovitz number $\mathrm{Ka}=\left(\delta_{F} / S_{L}\right)(\overline{\langle\varepsilon\rangle} / \nu)^{1 / 2}$ are increased from the first to the last case, whereas the Damköhler number $\mathrm{Da}=$ $\left(L_{11} / U^{\prime}\right) /\left(\delta_{F} / S_{L}\right)$ is decreased. Each of the four cases is characterized by the same $U^{\prime} / S_{L}$, but increasing $L_{11} / \delta_{F}$ is characterized by increasing $\mathrm{Re}_{t}$ and $\mathrm{Da}$, but decreasing $\mathrm{Ka}$. Thus, these 20 cases allow us to independently explore the influence of (i) $U^{\prime} / S_{L}$ and (ii) $L_{11} / \delta_{F}$ (or $\mathrm{Re}_{t}$ ) on turbulent reaction waves.

However, variations in $L_{11} / \delta_{F}$ and $\mathrm{Re}_{t}$ in the basic cases are linked, with all these 20 reaction waves being characterized by different Da or Ka. Accordingly, to gain insight into effects caused by variations in $\mathrm{Re}_{t}$ under conditions of constant $U^{\prime} / S_{L}, L_{11} / \delta_{F}$, and Da, cases $05-21-4 \delta$ and $10-21-4 \delta$, see Table III, are designed to be counterparts of the basic cases 05-21 and 10-21, respectively. For this purpose, the thickness $\delta_{F}$ is increased by a factor of about 3.3 by changing $\tau_{R}$ and $\mathcal{D}$ in Eq. (4). This factor is less than four, because $L_{11} / L_{y}=0.13$ and 0.11 at $\operatorname{Re}_{0}=50$ (cases 05-21 and 10-21) and $\operatorname{Re}_{0}=200$

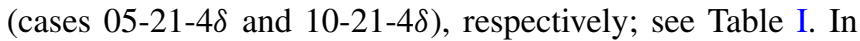
addition, (i) case 10-21-4 $-\mathrm{Ze}$, which is counterpart of case $10-21-4 \delta$, and (ii) cases 10-21* and 10-21*-Ze are set to explore the influence of $\mathrm{Ze}$ on the computed results. Moreover, a comparison of results obtained in cases 10-21 and 10-21* offers an opportunity to assess the sensitivity of numerical data to mesh resolution, because the mesh size $N_{x}$ is larger by a factor of four in the latter case; see the bottom line 
TABLE III. Other DNS cases.

\begin{tabular}{|c|c|c|c|c|c|c|c|c|c|c|c|}
\hline Case & $\operatorname{Re}_{0}$ & $\kappa_{f} / \kappa_{0}$ & $L_{11} / L_{y}$ & $\mathrm{Ze}$ & $U^{\prime} / S_{L}$ & $L_{11} / \delta_{F}$ & $\mathrm{Da}$ & $\mathrm{Ka}$ & $\delta_{F} / \eta$ & $\bar{\delta}_{T} / L_{11}$ & $\bar{S}_{T} / U^{\prime}$ \\
\hline $05-21-4 \delta$ & 200 & 3 & 0.11 & 6.0 & 0.5 & 2.1 & 4.1 & 1.8 & 12.3 & 1.52 & 2.19 \\
\hline $10-21-4 \delta$ & 200 & 3 & 0.11 & 6.0 & 10.0 & 2.1 & 0.2 & 36.2 & 12.3 & 5.67 & 0.42 \\
\hline $10-21-4 \delta-\mathrm{Ze}$ & 200 & 3 & 0.11 & 17.1 & 10.0 & 2.1 & 0.2 & 36.2 & 12.3 & 5.21 & 0.41 \\
\hline $10-21^{*}$ & 50 & 3 & 0.13 & 6.0 & 10.0 & 2.1 & 0.2 & 27.7 & 5.9 & 4.89 & 0.41 \\
\hline $10-21 *-\mathrm{Ze}$ & 50 & 3 & 0.13 & 17.1 & 10.0 & 2.1 & 0.2 & 27.7 & 5.9 & 5.15 & 0.41 \\
\hline $10-40-L_{11} / L$ & 200 & 6 & 0.11 & 6.0 & 10.0 & 4.0 & 0.4 & 16.1 & 4.5 & 5.28 & 0.58 \\
\hline $10-G$ & 200 & 3 & 0.06 & $\infty$ & 10.0 & $\infty$ & $\infty$ & 0 & 0 & 5.18 & 1.90 \\
\hline
\end{tabular}

in Table I. Furthermore, as already noted, comparison of results computed in the basic case 10-37 and 10-40- $L_{11} / L$ (see the next to the last lines in Tables I and III, respectively) offers an opportunity to gain insight into eventual influence of the width of the computational domain on the obtained results. Finally, simulations with $\mathrm{Re}_{0}=200, \kappa_{f} / \kappa_{0}=3$, and $U^{\prime} / S_{L}=10$ were also performed by solving Eq. (5), see case $10-G$ in Table III, in order to check consistency of the previous [24] and present simulations.

All in all, the present DNS study addresses turbulent reaction waves, which are characterized by significantly different Damköhler $(0.2 \leqslant \mathrm{Da} \leqslant 25.1)$ and Karlovitz $(0.4 \leqslant \mathrm{Ka} \leqslant$ 36.2) numbers and, therefore, are associated with different regimes of the wave propagation, e.g., "wrinkled flamelet" $\left(U^{\prime}<S_{L}, \mathrm{Da}>1\right.$, and $\left.\mathrm{Ka}<1\right)$ and "thin reaction zone" $(\mathrm{Ka}>1)$ regimes of premixed combustion [1].

It is worth stressing that, in the present work, certain scaling relations widely used in combustion literature, e.g., $\mathrm{Re}_{t} \propto$ $\left(U^{\prime} L_{11}\right) /\left(S_{L} \delta_{F}\right)$ and $\mathrm{Ka}^{2} \mathrm{Da}^{2} \propto \mathrm{Re}_{t}$, do not hold, because variations in $S_{L}$ and $\delta_{F}$ result not only from variations in the time scale $\tau_{R}$ but also from variations in the diffusivity $\mathcal{D}$, with the kinematic viscosity retaining the same value in all simulations. Thus, in order to vary a ratio of $L_{11} / \delta_{F}$ by retaining the same $U^{\prime} / S_{L}$ and the same turbulence characteristics, the Schmidt number $\nu / \mathcal{D}$ is varied.

By processing a profile of $c_{L}(x)$, obtained from a planar laminar wave, e.g., see Fig. 2, several wave thicknesses can be introduced in addition to $\delta_{F}$ used above. In particular, first, $\delta_{L}=1 / \max (|\nabla c|)$ characterizes the thickness of a zone associated with significant variations in $c_{L}(x)$ and a similar quantity is widely used to evaluate laminar flame thickness. Second, $\delta_{r}=\left|x\left(c_{2}\right)-x\left(c_{1}\right)\right|$ characterizes thickness of a reaction zone and is equal to distance between points $x\left(c_{1}\right)$ and $x\left(c_{2}\right)$ such that $c_{1}<c_{2}$ and $W\left(c_{1}\right)=W\left(c_{2}\right)=W\left(c^{*}\right) / 2$, where $c^{*}$ is associated with the peak $W^{*}=W\left(c^{*}\right)=\max \{W(c)\}$. Third, the reaction-zone thickness can be characterized by another length scale $\delta_{F}^{r}=\delta_{r} /\left(c_{2}-c_{1}\right)$ or by inverse gradient $\left.\left\langle|\nabla c|^{-1}\right\rangle\right|_{c_{1}<c<c_{2}}=\delta_{r}^{-1} \int_{x\left(c_{1}\right)}^{x\left(c_{2}\right)}\left|\frac{\partial}{\partial_{x}} c_{L}\right|^{-1} d x$ averaged of the zone. Under conditions of the present study, $\delta_{L}=1.3 \delta_{F}$, $\delta_{r}=0.28 \delta_{F}, \quad \delta_{F}^{r}=2.2 \delta_{F}$, and $\left.\left\langle|\nabla c|^{-1}\right\rangle\right|_{c_{1}<c<c_{2}}=3.3 \delta_{F}$ if $\mathrm{Ze}=17.1$ (cases 10-21-4 $\delta$-Ze and 10-21 ${ }^{*}$-Ze) or $\delta_{L}=1.7 \delta_{F}$, $\delta_{r}=0.72 \delta_{F}, \delta_{F}^{r}=2.5 \delta_{F}$, and $\left.\left\langle|\nabla c|^{-1}\right\rangle\right|_{c_{1}<c<c_{2}}=3.1 \delta_{F}$ if $\mathrm{Ze}=6$ (other cases).

Using a resolution of four grid cells per $\delta_{F}$ (this resolution was used in all cases in Table II characterized by $\mathrm{Ze}=6$ ), the inner reaction zone $\left(\delta_{r}\right)$ is resolved with around three grid points. Such a resolution (four cells per $\delta_{F}$ ) was used in recent $2 \mathrm{D}$ simulations of development of the hydrodynamic instability [40,41] of a laminar premixed flame in a wide computational domain [42] and was shown to be sufficient in that case. The fact that this resolution is sufficient for the goals of the present study is also confirmed by good agreement between DNS results obtained in cases 10-21 and 10-21*; see the last two columns in Tables II and III. Moreover, comparison of results shown in solid and dashed lines in Fig. 2(b) indicates that the used numerical grids allow us to resolve well not only smooth spatial variations in $c$ but also spatial variations in the molecular diffusion and reaction terms in Eq. (3).
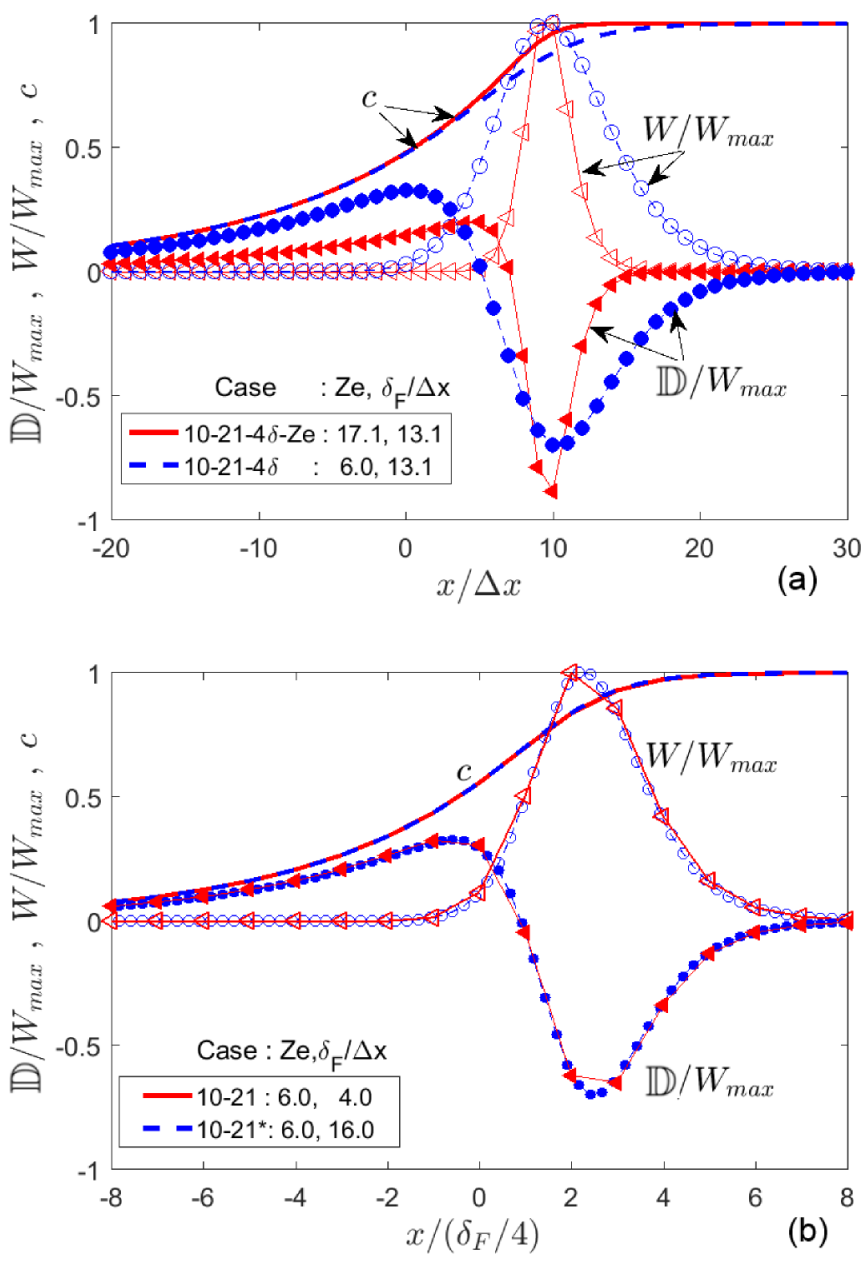

FIG. 2. Profiles of $c$, normalized molecular transport and reaction terms, obtained in 1D simulations of laminar waves. (a) Effect of the Zeldovich number Ze, with spatial resolution being the same

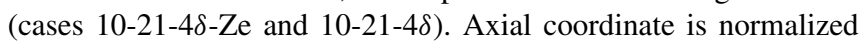
using $\Delta x$. (b) Effect of spatial resolution, with Ze being the same (cases 10-21 and 10-21*). Axial coordinate is normalized using $\delta_{F} / 4$. 
In the first three $(05-21-4 \delta, 10-21-4 \delta$, and 10-21$4 \delta$-Ze) and the next two $\left(10-21^{*}\right.$ and $\left.10-21^{*}-4 \delta\right)$ cases in Table III, a ratio of $\delta_{F} / \Delta x$ is larger (13 and 16, respectively), because either $\delta_{F}$ is larger, but $\Delta x$ is standard (the three cases characterized by $\operatorname{Re}_{0}=200$ ), or $\Delta x$ is smaller (cases $10-21^{*}$ and $10-21 *-4 \delta$ ).

In order to study a fully developed reaction wave, a planar wave $c(\boldsymbol{x}, t=0)=c_{L}(\xi)$ was initially $(t=0)$ released at $x^{0}=$ $L_{x} / 2$ such that $\int_{-\infty}^{0} c_{L}(\xi) d \xi=\int_{0}^{\infty}\left[1-c_{L}(\xi)\right] d \xi$ and $\xi=$ $x-x^{0}$. Here, $c_{L}(\xi)$ is the precomputed laminar-wave profile. Subsequently, evolution of this field $c(\boldsymbol{x}, t)$ was simulated by solving Eq. (3). To enable periodic propagation of $c$ field along the $x$ direction, the field is extrapolated outside the axial boundaries of the computational domain at each time step $t^{n}$ as follows: $c\left(x^{\prime}, y, z, t^{n}\right)=c\left(x, z, t^{n}\right)$, where $x^{\prime}=x+I L_{x}$ and $I$ is an arbitrary (positive or negative) integer number. Subsequently, Eq. (6) is solved at $x^{\prime} \in\left[\zeta\left(t^{n}\right)-\Delta, \zeta\left(t^{n}\right)+\Delta\right]$, where $\zeta\left(t^{n}\right)$ is the mean coordinate of a reaction wave on the $x^{\prime}$ axis and $\Delta=0.45 L_{x}$ in order to avoid numerical artifacts in the vicinity of $x^{\prime}=\zeta\left(t^{n}\right) \pm 0.5 L_{x}$. In two remaining sections, i.e., $x^{\prime} \in\left[\zeta\left(t^{n}\right)-0.5 L_{x}, \zeta\left(t^{n}\right)-\Delta\right]$ and $x^{\prime} \in\left[\zeta\left(t^{n}\right)+\Delta, \zeta\left(t^{n}\right)+0.5 L_{x}\right]$, the scalar $c\left(t^{n}\right)$ is set equal to zero (fresh reactants) and unity (products), respectively, because the entire flame brush is always kept within the interval of $x^{\prime} \in\left[\zeta\left(t^{n}\right)-\Delta, \zeta\left(t^{n}\right)+\Delta\right]$ in the present simulations. Finally, the obtained solution $c\left(x^{\prime}, y, z, t^{n}\right)$ is translated back to the $x$ coordinate. ${ }^{3}$

Computations of fully developed statistics with sampling every $100 \Delta t$ were started after the forced turbulence reached statistical stationarity at $t=t_{*}=6000 \Delta t>3.5 \tau_{t}^{0}$. Here, $\tau_{t}^{0}=\Lambda_{0} / U^{\prime}$ is the initial turbulence eddy turn over time. In all cases, the total sampling duration was larger than $50 \tau_{t}^{0}$. It is worth remembering that the statistically stationary forced turbulence was characterized by $0.25 \tau_{t}^{0} \leqslant \tau_{t}<0.8 \tau_{t}^{0}$; see Table I.

Statistics were sampled using the following methods. First, for each characteristic $q(\boldsymbol{x}, t)$ of the reaction wave $c(\boldsymbol{x}, t)$, its statistically stationary mean value $\bar{q}(x)$ was evaluated by averaging the DNS data over transverse ( $y$ and $z$ ) coordinates and over time at $t>t_{*}$. Second, the $x$ coordinate was mapped to a $\bar{c}(x)$ coordinate to yield $\bar{q}(\bar{c})$, as discussed in detail in Ref. [24].

Moreover, when analyzing the DNS data in the present paper, we will deal with a few ${ }^{4}$ quantities $q$ conditioned to the reaction zone, i.e., $c_{1}<c<c_{2}$ such that $W\left(c_{1}\right)=W\left(c_{2}\right)=$ $\max \{W(c)\} / 2$, at various $\bar{c}(x)$. Such conditioned quantities are found using 3D probability density functions (PDFs) $\bar{P}(q, c, \bar{c})$ and the following expressions:

$$
\begin{aligned}
\overline{\langle q\rangle_{f}}(\bar{c})= & \int_{-\infty}^{+\infty} \int_{c_{1}}^{c_{2}} q \bar{P}(q, c, \bar{c}) d c d q / \\
& \int_{-\infty}^{+\infty} \int_{c_{1}}^{c_{2}} \bar{P}(q, c, \bar{c}) d c d q .
\end{aligned}
$$

\footnotetext{
${ }^{3}$ See texts around Eq. (3.3) in Ref. [24] for a more detailed description of the implementation of the $x$ periodicity.

${ }^{4}$ Similar data obtained for a wide set of other, more than 15 , local characteristics of reaction wave and turbulent flow will be analyzed in subsequent publications.
}

It is worth noting that, in order to improve the statistical convergence of reported results, the $3 \mathrm{D}$ PDFs $\bar{P}(q, c, \bar{c})$ are evaluated in the run-time regime; i.e., each grid cell and each sampling time step contribute to the PDF.

More specifically, the 3D PDFs are determined as follows. First, boundaries $q_{\min }$ and $q_{\max }$ of expected variations of a quantity $q(\boldsymbol{x}, t)$ are found in a test prerun and an interval bounded by $q_{\min }$ and $q_{\max }$ is uniformly cut into 100 discrete bins. Second, an interval of $0 \leqslant c(\boldsymbol{x}, t) \leqslant 1$ is uniformly cut into 100 discrete bins. Subsequently, at each time step $t^{n}$ of the simulations, (i) negligible values of probabilities of $q<q_{\text {min }}$ and $q>q_{\max }$ are checked and (ii) the set of $q(\boldsymbol{x}, t), c(\boldsymbol{x}, t)$, and $x_{i}=i \Delta x$ is sampled over the entire computational domain; i.e., if the values of $q\left(x_{i}, y_{j}, z_{k}, t^{n}\right)$ and $c\left(x_{i}, y_{j}, z_{k}, t^{n}\right)$ belong to the $l$ th $q$ bin and $m$ th $c$ bin, respectively, then unity is added to the $\{l, m, i\}$-th element of the 3D $q$-sampling array. Finally, at the postprocessing stage, $\bar{P}(q, c, \bar{c})$ is obtained by (i) renormalizing all elements of the $3 \mathrm{D}$ sampling array in order for their sum to be equal to unity and (ii) translating the $x$ dependence into $\bar{c}$ dependence using the monotonous function $\bar{c}(x)$.

\section{RESULTS AND DISCUSSION}

The 3D images of the reaction zone surface, obtained in the present DNS, look similar to the 3D images of an infinitely thin self-propagating interface, obtained by solving Eq. (5); see Fig. 3 in Yu et al. [24]. To provide insight into the structure of the reaction zone in the present DNS, typical instantaneous $W[c(\boldsymbol{x}, t)]$ fields are shown in Fig. 3. In case 05-67 (bottom row), characterized by the lowest $\mathrm{Ka}$, the reaction zone is weakly perturbed and moderately wrinkled, with distance between isosurfaces of $c(\boldsymbol{x}, t)=c_{1}$ and $c(\boldsymbol{x}, t)=c_{2}$ being weakly varied. In case 10-67 (top row), characterized by a high $\mathrm{Ka}=11.0$ and a low $\mathrm{Da}=0.7$, the reaction zone is still predominantly thin, but is strongly wrinkled. In cases $10-21-4 \delta$ and $10-21-4 \delta-Z e$ (two middle rows), characterized by the highest $\mathrm{Ka}=36.2$ and the lowest $\mathrm{Da}=0.2$, substantial perturbations of the reaction zone are clearly visible, with both distributed and sufficiently thin reaction zones being simultaneously observed in different spatial regions.

Figure 3 is consistent with contemporary knowledge on the structure of reaction zones in highly turbulent premixed flames. First, as reviewed elsewhere $[3,43]$, recent experimental and DNS data indicate that reaction zones remain thin and retain their laminar structure even in intense turbulence associated with high Ka. Images reported in top and bottom rows in Fig. 3 are qualitatively consistent with such data. Second, 2D images of reaction zones, obtained in a few very recent experimental studies [44-46] of premixed turbulent flames characterized by very high $\mathrm{Ka}$, showed that such "zones exhibited regions of both relatively thin and distributed reactions" [44] (p. 4089). A similar behavior of reaction zones is shown in the second row in Fig. 3. Recent DNS of premixed flames in very intense turbulence also indicated transition from thin to distributed reaction zones at very high $\mathrm{Ka}[10,47]$.

However, values of $\mathrm{Ka}$ associated with such a transition in the present simulations are significantly lower than the counterpart values of Ka reported in the cited experimental and DNS papers. This quantitative difference can be attributed 

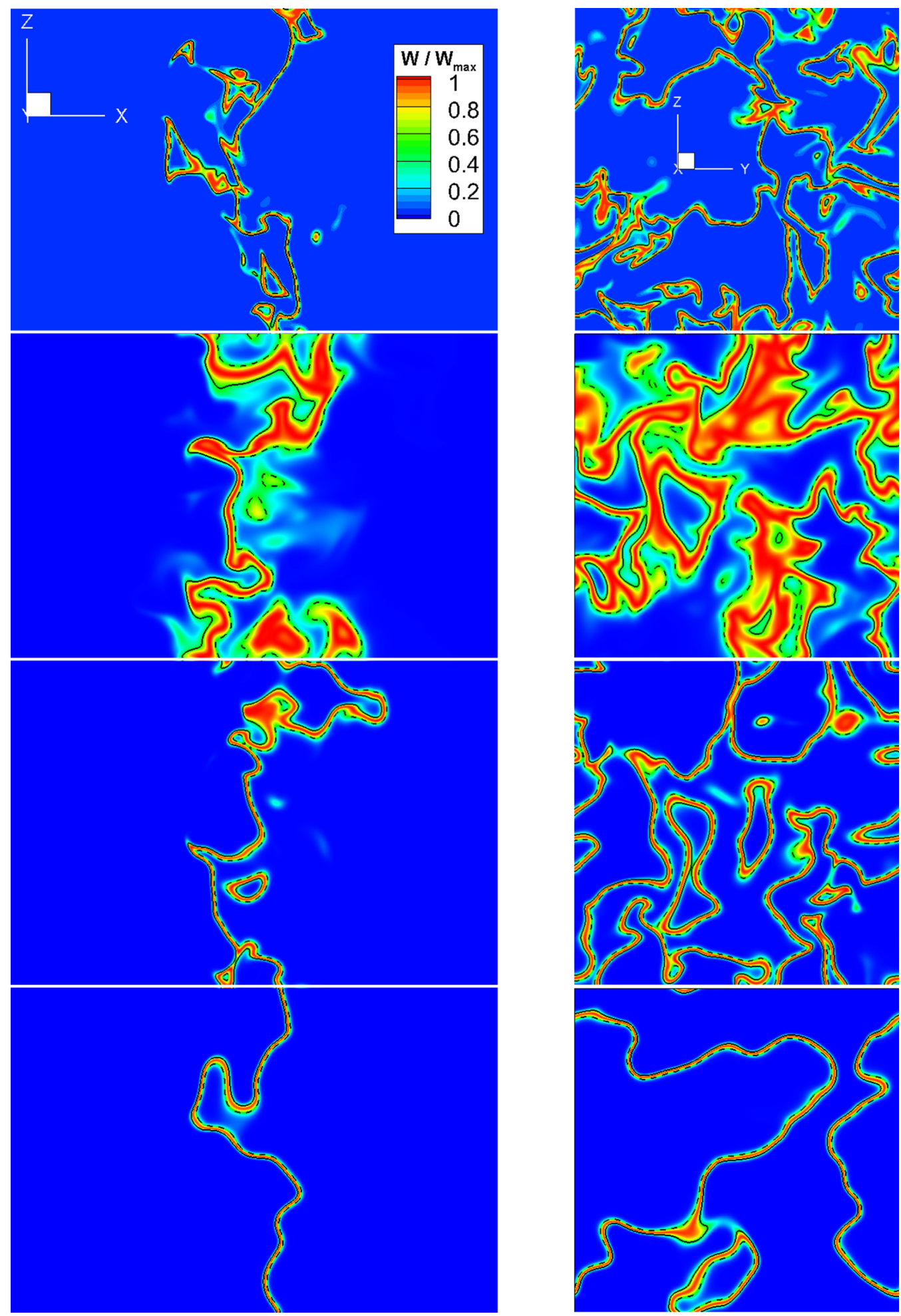

FIG. 3. Typical 2D images of the normalized reaction rate $W(c) / \max \{W(c)\}$ in $y$-slice planes (left column) and $x$-slice planes (right column). The rate is normalized using the maximum value $\max \{W(c)\}$ of function $W(c)$ given by Eq. (4) provided that $\tau$, $\tau_{R}$, and Ze are kept constant. Black dashed and solid lines show boundaries of the reaction zone, i.e., $c(\boldsymbol{x}, t)=c_{1}$ and $c_{2}$, respectively. All cases are characterized by $\operatorname{Re}_{0}=200$, but different $U^{\prime} / S_{L}=10, L_{11} / \delta_{F}=6.7, \mathrm{Da}=0.7$, and $\mathrm{Ka}=11.0$ (case 10-67, top row) $U^{\prime} / S_{L}=10, L_{11} / \delta_{F}=2.1, \mathrm{Da}=0.2$, and $\mathrm{Ka}=36.2$ (cases 10-21-4 $\delta$ and 10-21-4 $\delta$-Ze, two middle rows); $U^{\prime} / S_{L}=0.5, L_{11} / \delta_{F}=6.7, \mathrm{Da}=13.5$, and $\mathrm{Ka}=0.6$ (case 05-67, bottom row).

to the lack of thermal expansion effects in the present computations. In the case of premixed combustion, small-scale eddies are known to be inefficient in wrinkling reaction-zone surface $[48,49]$, in particular, because they rapidly disappear due to dilatation and an increase in viscosity within the flame preheat zone. However, because these two phenomena 
vanish in a constant-density flow, reaction waves do not destroy the smallest-scale turbulent eddies and, therefore, do not reduce the highest local rates of stretch of reaction zones under conditions of the present DNS. Accordingly, in a constant-density turbulent flow, an "efficient" Ka experienced by a reaction wave appears to be significantly higher than an "efficient" Ka experienced by a premixed flame in statistically the same incoming flow of unburned reactants.

\section{A. Mean characteristics of turbulent reaction wave}

It is worth remembering that the key difference between the present and previous [24] DNSs consists in substituting Eq. (5) with Eq. (3), which describe propagation of an infinitely thin reaction zone and a reaction zone of a nonzero thickness, respectively. Therefore, in order to gain insight into the influence of the reaction zone thickness on the speed and structure of the mean reaction wave, exactly the same quantities were extracted from the previous [24] and present DNS databases, followed by comparison of these quantities. Accordingly, the structure of the present subsection is as follows. In Sec. IV A 1, we will briefly summarize results that are basically similar in the two DNS series. In Sec. IV A 2, we will report and discuss a significant effect of the reaction zone thickness on the mean reaction wave speed.

\section{Mean wave thickness and turbulent diffusivity}

This section is restricted to a summary of results that are similar to results obtained by Yu et al. [24] by solving Eq. (5). The reader interested in a detail presentation of such results is referred to Secs. 4.2-4.4 and Figs. 6-16 in the cited paper.

Let us consider fully developed mean wave brush thickness evaluated as follows:

$$
\bar{\delta}_{T}=\frac{1}{\max |\nabla \bar{c}|} .
$$

Values of $\bar{\delta}_{T}$ normalized using $L_{11}$ are reported in the next to the right column in Tables II and III. Comparison of cases 1021 and 10-21* in Tables II and III, respectively, indicates that an increase in spatial resolution of the local wave thickness by

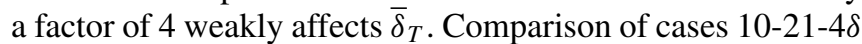
and $10-21-4 \delta$-Ze or $10-21^{*}$ and $10-21^{*}-$ Ze in Table III shows that an increase in Ze by a factor of about 3 weakly affects $\bar{\delta}_{T}$. Accordingly, in the rest of the present section, discussion will be restricted to cases characterized by the same $\mathrm{Ze}=6.0$.

Fully developed $\bar{\delta}_{T}$ obtained in these cases are plotted in black symbols in Fig. 4(a), whereas the counterpart red symbols show results obtained earlier [24] by tracking an infinitely thin self-propagating interface in statistically the same turbulence. Comparison of the black and red symbols indicates that a nonzero thickness of a reaction wave weakly affects fully developed $\bar{\delta}_{T}$.

In order to explore dependence of the computed $\bar{\delta}_{T}$ on basic mixture and turbulence characteristics, the fits

$$
Y=a X^{b}
$$

were applied to the present DNS data obtained in 20 basic cases, see Table II, and cases $05-21-4 \delta$ and $10-21-4 \delta$, see Table III. Here, $Y=\left\{\bar{\delta}_{T} / L_{11}, \bar{\delta}_{T} / \delta_{F},\left(\bar{\delta}_{T}-\delta_{L}\right) / L_{11},\left(\bar{\delta}_{T}-\right.\right.$ $\left.\left.\delta_{L}\right) / \delta_{F}\right\}$ and $X=\left\{U^{\prime} / S_{L}, \mathrm{Da}, \mathrm{Ka}, \mathrm{Da} \mathrm{Ka},\left(U^{\prime} / S_{L}\right)\left(L_{11} / \delta_{F}\right)^{q}\right\}$.
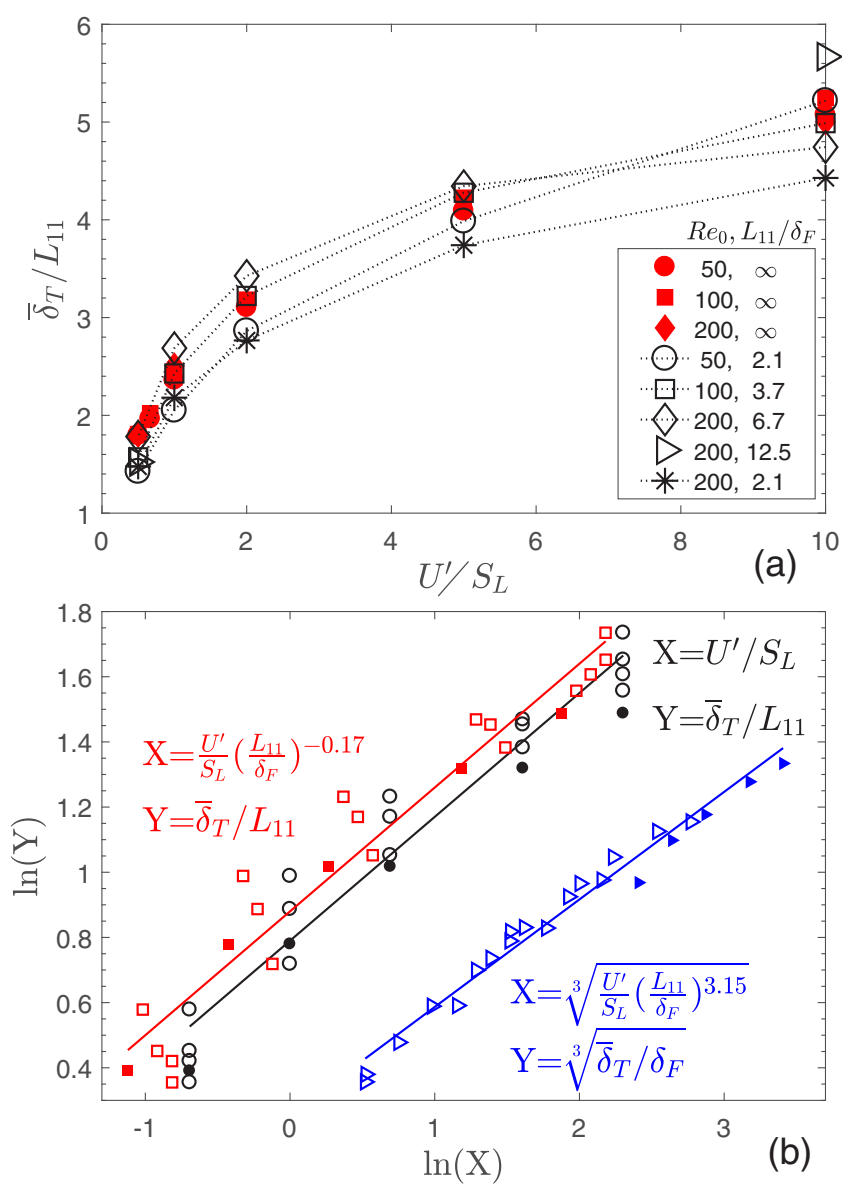

FIG. 4. Fully developed mean turbulent wave thickness. (a) $\bar{\delta}_{T} / L_{11}$ vs $U^{\prime} / S_{L}$. DNS data obtained in cases (i) $05-21$ to 10 $21\left(\operatorname{Re}_{0}=50\right)$, (ii) $05-37$ to $10-37\left(\operatorname{Re}_{0}=100\right)$, (iii) 05-67 to $10-67\left(\operatorname{Re}_{0}=200\right)$, (iv) $05-125$ to $10-125$, see Table II, and (v) $05-21-4 \delta$ and $10-21-4 \delta$, see Table III, are shown in black (i) circles, (ii) squares, (iii) diamonds, (iv) stars, and (v) triangles, respectively. Filled red circles, triangles, and diamonds show results obtained earlier [24] by tracking an infinitely thin self-propagating interface in statistically the same turbulence generated at (i) $\mathrm{Re}_{0}=50$, (ii) $\mathrm{Re}_{0}=100$, and (iii) $\mathrm{Re}_{0}=200$, respectively. (b) Best fits to the present DNS data; $X=U^{\prime} / S_{L}$ and $Y=\bar{\delta}_{T} / L_{11}$ (black circles and line), $X=\left(U^{\prime} / S_{L}\right)\left(L_{11} / \delta_{F}\right)^{-0.17}$ and $Y=\bar{\delta}_{T} / L_{11}$ (red squares and line), $X=\left(U^{\prime} / S_{L}\right)\left(L_{11} / \delta_{F}\right)^{3.15}$ and $Y=\bar{\delta}_{T} / \delta_{F}$ (blue triangles and line, which show $\ln (Y) / 3$ vs $\ln (X) / 3$ ). Filled symbols show data obtained in five cases associated with $\kappa_{f} / \kappa_{0}=1$; see Table II. Open symbols show data obtained in the other 17 cases.

It is worth remembering that the Schmidt number $v / \mathcal{D}$ was varied in the present study in order to vary $L_{11} / \delta_{F}$ by retaining the same $U^{\prime} / S_{L}$ and the same turbulence characteristics. Therefore, a function of $U^{\prime} / S_{L}$ and $L_{11} / \delta_{F}$ is not reduced to a function of $\mathrm{Da}$ and $\mathrm{Ka}$.

When applying Eq. (11) to the present data, parameters $a$ and $b$ were calculated using least square fit in logarithmic coordinates. A coefficient of determination

$R^{2}=1-\left[\sum_{j=1}^{N}\left(Y_{j}-a X_{j}^{b}\right)^{2}\right]\left[\sum_{j=1}^{N}\left(Y_{j}-\frac{1}{N} \sum_{k=1}^{N} Y_{k}\right)^{2}\right]^{-1}$ 
TABLE IV. Results of fitting the DNS data on $\bar{\delta}_{T}$ : 17 cases are characterized by $\kappa_{f} / \kappa_{0}=3$ and $L_{11} / L \approx 0.12$, and 22 cases include five more cases characterized by $\kappa_{f} / \kappa_{0}=1$ and $L_{11} / L=0.2$.

\begin{tabular}{|c|c|c|c|c|c|c|c|c|c|}
\hline \multirow[b]{2}{*}{$Y$} & \multirow[b]{2}{*}{$X$} & \multicolumn{4}{|c|}{22 cases } & \multicolumn{4}{|c|}{17 cases } \\
\hline & & $q$ & $\ln a$ & $b$ & $R^{2}$ & $q$ & $\ln a$ & $b$ & $R^{2}$ \\
\hline$\frac{\overline{\delta_{T}}}{L_{11}}$ & $\frac{U^{\prime}}{S_{L}}$ & & 0.79 & 0.38 & 0.93 & & 0.80 & 0.39 & 0.95 \\
\hline$\frac{\bar{\delta}_{T}}{L_{11}}$ & $\frac{U^{\prime}}{S_{L}}\left(\frac{L_{11}}{\delta_{F}}\right)^{q}$ & -0.17 & 0.88 & 0.38 & 0.94 & 0.02 & 0.79 & 0.39 & 0.95 \\
\hline$\frac{\bar{\delta}_{T}}{\delta_{F}}$ & $\frac{U^{\prime}}{S_{L}}\left(\frac{L_{11}}{\delta_{F}}\right)^{q}$ & 3.15 & 0.77 & 0.33 & 0.96 & 3.76 & 0.60 & 0.32 & 0.96 \\
\hline
\end{tabular}

was also evaluated. When using $X=\left(U^{\prime} / S_{L}\right)\left(L_{11} / \delta_{F}\right)^{q}$, the exponent $q$ was varied from -4 to 4 with a step of 0.01 and the value of $q$ that yielded the highest $R^{2}$ was selected.

Parameters of three best fits characterized by $R^{2}>0.90$ are listed in Table IV, with these fits being plotted in Fig. 4(b). It is worth noting that results calculated for $\bar{\delta}_{T}-\delta_{L}$ are not reported in Table IV, because subtraction of $\delta_{L}$ from $\bar{\delta}_{T}$ reduces $R^{2}$, i.e., impedes fitting, for all $X$. Moreover, approximations of (i) 17 DNS cases characterized by $\kappa_{f}=\kappa_{0}=3$ and $L_{11} / L \approx$ 0.12 , see open symbols in Fig. 4(b), and (ii) 22 DNS cases, which include five more cases characterized by $\kappa_{f}=\kappa_{0}=1$ and $L_{11} / L=0.2$, see filled symbols in Fig. 4(b), are close to one another, thus implying a minor influence of the relative width $L / L_{11}$ of the computational domain on the simulated $\bar{\delta}_{T}$.

Table IV shows that $\bar{\delta}_{T}$ is substantially increased by $U^{\prime} / S_{L}$, is almost proportional to $L_{11}$, and depends weakly on $\delta_{F}$. As discussed by $\mathrm{Yu}$ et al. [24], such a dependence of fully developed mean flame brush thickness on $U^{\prime} / S_{L}$ has never been predicted by a model of premixed turbulent combustion. It is worth noting, however, that expressions obtained by Klimov [50] yield $\delta_{T, \infty} \propto L_{11}\left(U^{\prime} / S_{L}\right)^{q}$ with $q \approx 0.5$. This power exponent is not very far from $b \approx 0.4$ reported for $\bar{\delta}_{T} / L_{11}$ as a function of $U^{\prime} / S_{L}$ in Table IV.

Let us consider fully developed turbulent diffusivity evaluated as follows:

$$
\bar{D}_{T} \equiv-\frac{\overline{u^{\prime} c^{\prime}}}{\nabla_{x} \bar{c}} .
$$

Similar to results computed by Yu et al. [24] by solving the $G$ equation, Eq. (5), $\bar{D}_{T}$ obtained in the present DNS (i) is always positive, in line with the concept of turbulent diffusion, (ii) depends weakly on $\bar{c}$, but (iii) is reduced by $S_{L} / U^{\prime}$; see Fig. 5.

Due to weak dependence of $\bar{D}_{T}$ on $\bar{c}$, Yu et al. [24] suggested characterizing the influence of chemical reactions on turbulent diffusivity using a single averaged diffusivity,

$$
\overline{D_{T}^{*}} \equiv \int_{0}^{1} \bar{D}_{T} d \bar{c}=-\int_{0}^{1} \frac{\overline{u^{\prime} c^{\prime}}}{\nabla_{x} \bar{c}} d \bar{c}=-\int_{0}^{L_{x}} \overline{u^{\prime} c^{\prime}} d x .
$$

In line with the DNS study by Yu et al. [24], $\overline{D_{T}^{*}}$ depends weakly on $L_{11} / L$, which was varied by varying either $\operatorname{Re}_{0}$ or $\kappa_{f} / \kappa_{0}$ in the present study, and is decreased with decreasing $U^{\prime} / S_{L}$; see Fig. 6(a). Figure 6(b) shows a substantial reduction of the magnitude of the effect due to substitution of the length scale $L_{11}$ with the thickness $\bar{\delta}_{T}$ when normalizing $\bar{D}_{T}$.

A decrease in turbulent diffusivity due to chemical reactions in constant-density flows, shown in Fig. 6(a), is well known
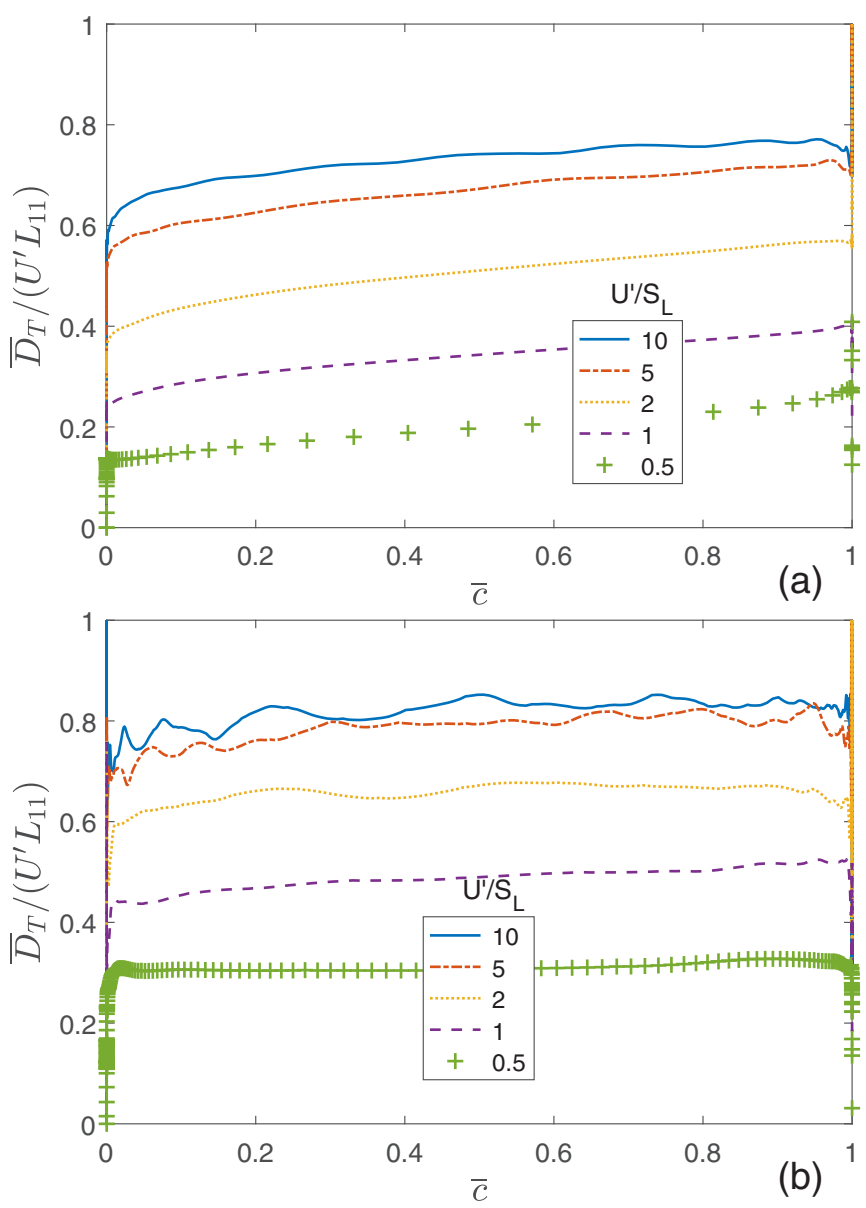

FIG. 5. Dependencies of normalized fully developed turbulent diffusivity $\bar{D}_{T} /\left(U^{\prime} L_{11}\right)$ evaluated using Eq. (13) on $\bar{c}$ obtained at (a) $L_{11} / L=2.1$ and (b) $L_{11} / L=6.7$.

after the seminal work by Corrsin [51]. For instance, such an effect results straightforwardly from transport equations for velocities conditioned to reactants or products [3,52]. Moreover, a theory that yields such an effect was recently developed [53]. The effect stems from inability of large-scale turbulent eddies to fully contribute to mixing if the reactant is consumed by a chemical reaction during the eddy lifetime [53]. Nevertheless, the effect is often ignored by the combustion community.

Comparison of open black and filled red symbols in Fig. 6(a) reveals only a slight influence of a nonzero thickness of reaction wave on $\overline{D_{T}^{*}} /\left(U^{\prime} L_{11}\right)$. The effect is less pronounced at higher $U^{\prime} / S_{L}$ at $\operatorname{Re}_{0}=50$, see circles, or at higher $\operatorname{Re}_{0}$ (other black symbols). However, scatter of the same DNS data reported in a form of $\bar{D}_{T}^{*} /\left(U^{\prime} \bar{\delta}_{T}\right)$ vs $U^{\prime} / S_{L}$ is increased due to the nonzero thickness, cf. open and filled symbols in Fig. 6(b), with the effect being more pronounced at lower $U^{\prime} / S_{L}$.

Finally, comparisons of $\bar{D}_{T} /\left(U^{\prime} L_{11}\right)=0.395$ and 0.384 , obtained in cases $10-21-4 \delta$ and $10-21-4 \delta$-Ze, respectively, or $\bar{D}_{T} /\left(U^{\prime} L_{11}\right)=0.366,0.379$, and 0.365 , evaluated in cases 10-21, 10-21*, and 10-21*-Ze, respectively, do not show a notable effect of either Ze or numerical resolution on the fully developed averaged diffusivity. 

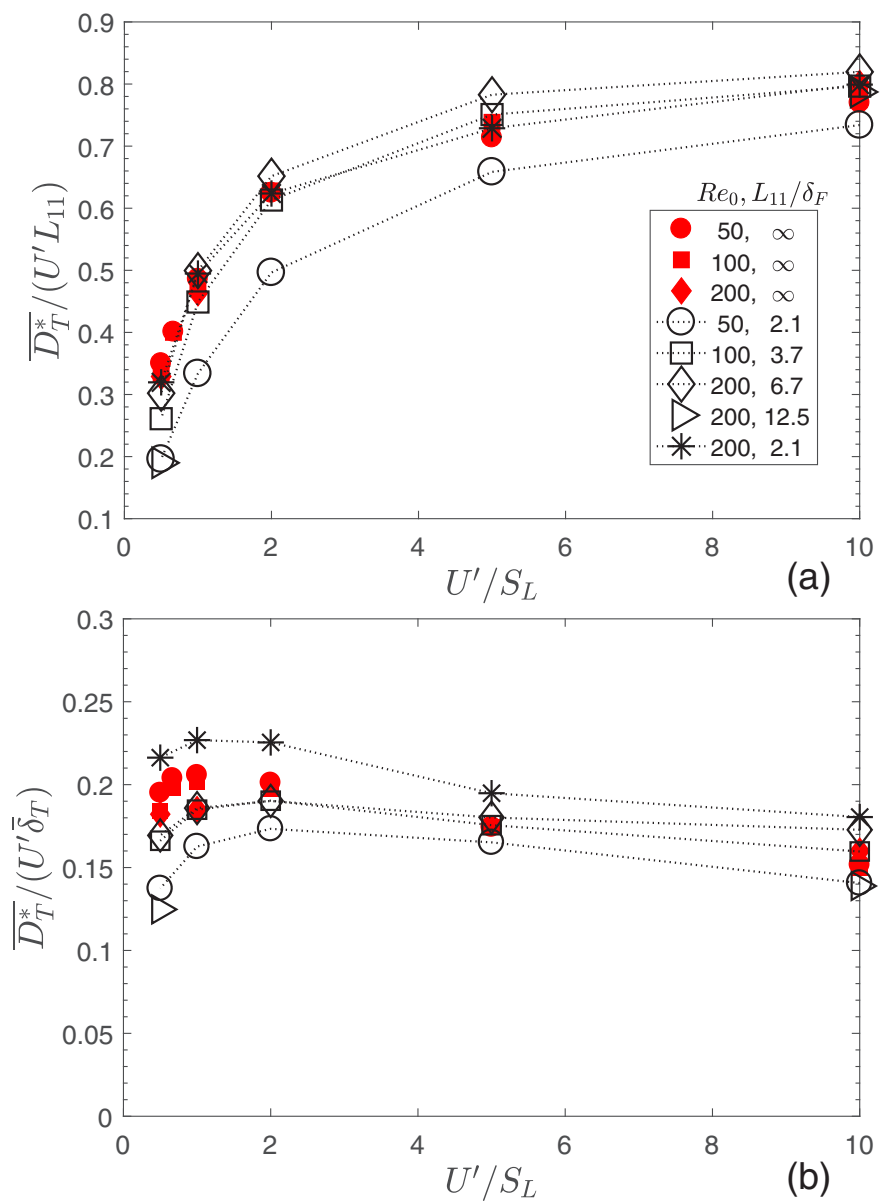

FIG. 6. Fully developed averaged diffusivity $\overline{D_{T}^{*}}$ normalized using (a) $U^{\prime} L_{11}$ and (b) $U^{\prime} \bar{\delta}_{T}$ vs $U^{\prime} / S_{L}$. Symbol legends are explained in caption to Fig. 4.

\section{Turbulent wave speed}

Fully developed turbulent wave speeds $\bar{S}_{T}$ were evaluated (i) by differentiating time dependencies of the axial coordinate $x_{f}(t)$ of a plane $x=x_{f}(t)$, where the value $\langle c\rangle$ of $c(\boldsymbol{x}, t)$, averaged over transverse coordinates, was equal to 0.5 , and (ii) by averaging the obtained $S_{T}(t)$ over time at $t>t_{*}$. Fully developed turbulent consumption velocities $\bar{U}_{T}$ were computed by averaging

$$
U_{T}(t)=\frac{1}{L_{y} L_{z}} \int_{0,0,0}^{L_{x}, L_{y}, L_{z}} W[c(\boldsymbol{x}, t)] d \boldsymbol{x}
$$

over time at $t>t_{*}$. Because the obtained values of $S_{T}(t)$ and $U_{T}(t)$ are always close to one another, we will address $\bar{S}_{T}$ in the rest of the paper.

Values of $\bar{S}_{T}$ normalized using $U^{\prime}$ are reported in the right columns in Tables II and III. Comparison of cases 10-21 and $10-21^{*}$ indicates that an increase in spatial resolution of the local wave thickness by a factor of four weakly affects $\bar{S}_{T}$. Comparison of cases $10-21-4 \delta$ and $10-21-4 \delta$-Ze or $10-21^{*}$ and $10-21^{*}-\mathrm{Ze}$, see Table III, shows that an increase in Ze by a factor of about three weakly affects $\bar{S}_{T}$. Accordingly, in the rest of the present subsection, discussion will be restricted to 20 basic cases, see Table II, and cases $05-21-4 \delta$ and $100-21-4 \delta$, see Table III, characterized by the same Ze.
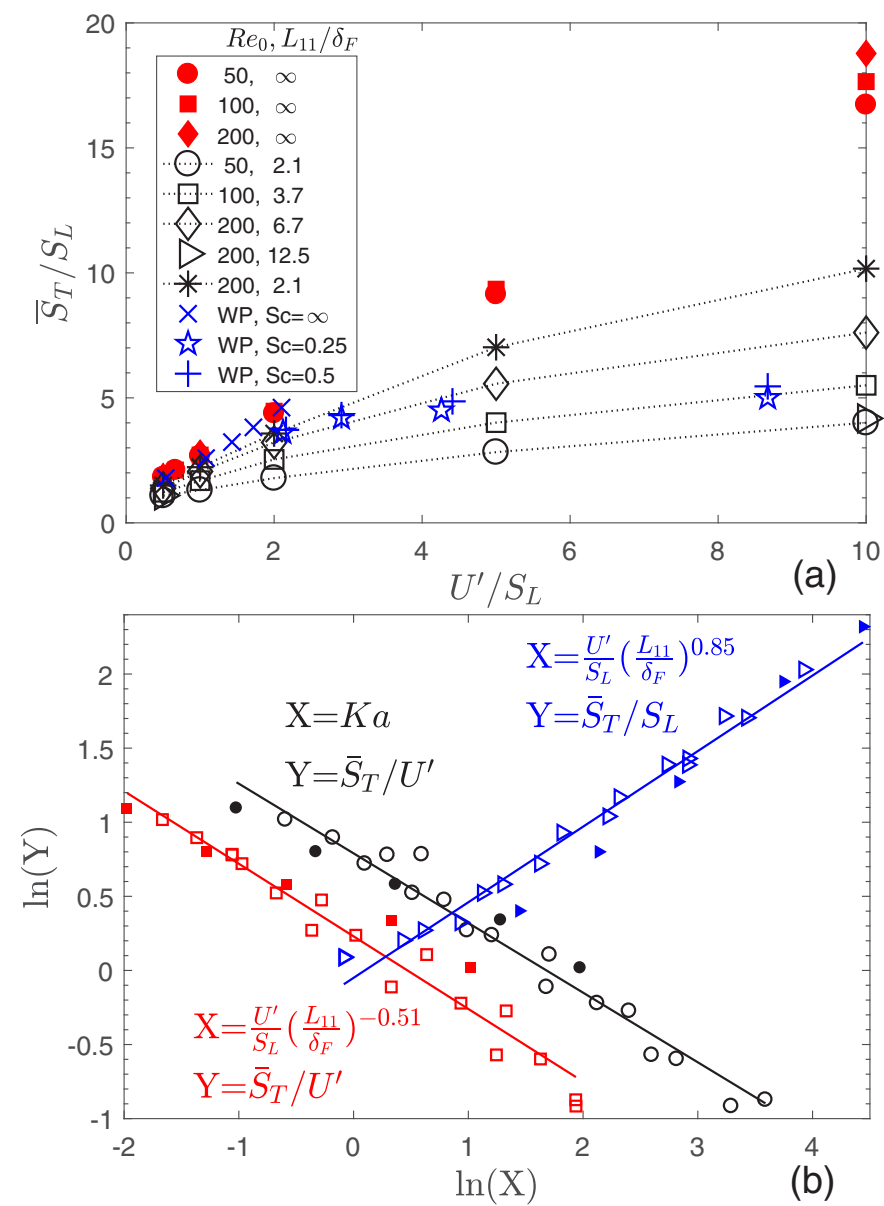

FIG. 7. Fully developed turbulent wave speeds. (a) $\bar{S}_{T} / S_{L}$ vs $U^{\prime} / S_{L}$. Blue crosses, pentagons, and pluses show DNS data computed by Wenzel and Peters [54,55] using $S c=\infty, 0.25$, and 0.50 , respectively. Other symbol legends are explained in caption to Fig. 4. (b) Best fits to the present DNS data; $X=\mathrm{Ka}$ and $Y=\bar{S}_{T} / U^{\prime}$ (black circles and line), $X=\left(U^{\prime} / S_{L}\right)\left(L_{11} / \delta_{F}\right)^{-0.51}$ and $Y=\bar{S}_{T} / U^{\prime}$ (red squares and line), $X=\left(U^{\prime} / S_{L}\right)\left(L_{11} / \delta_{F}\right)^{0.85}$, and $Y=\bar{S}_{T} / S_{L}$ (blue triangles and line). Filled symbols show data obtained in five cases associated with $\kappa_{f} / \kappa_{0}=1$; see Table II. Open symbols show data obtained in other 17 cases.

Black symbols in Fig. 7(a) show normalized fully developed mean wave speeds $\bar{S}_{T} / S_{L}$, computed in the present work. Filled red symbols show $\bar{S}_{T} / S_{L}$ simulated in our previous DNS study [24] of self-propagation of an infinitely thin front in statistically the same turbulence. Blue crosses, pentagons, and pluses show $\bar{S}_{T} / S_{L}$ obtained by Wenzel and Peters [54,55] in 3D DNS of self-propagation of an infinitely thin front in constant-density turbulence. These authors solved the $G$ equation (5) and allowed for the linear dependence $S_{L}=$ $S_{L}^{0}-v h /$ Sc of the local front speed $S_{L}$ on its local curvature $h=-\nabla \cdot(\nabla G /|\nabla G|)$. The sensitivity of the local front speed to its local curvature was varied by changing the Schmidt number Sc. The case of $S c=\infty$ corresponds to a constant $S_{L}$, i.e. this case is basically similar to the problem studied by $\mathrm{Yu}$ et al. [24].

Figure 7(a) shows that, first, a nonzero thickness of the reaction wave reduces $\bar{S}_{T} / S_{L}$ when compared to an infinitely 
thin front, cf. open black and filled red symbols. Second, $\bar{S}_{T} / S_{L}$ is increased by $L_{11} / \delta_{F}$, cf. black circles, squares, and diamonds. Third, in cases $10-21$ and $10-21-4 \delta$ or $05-21$ and 05-21-4 $\delta$, characterized by different $\operatorname{Re}_{t}$ or $\mathrm{Ka}$, but the same $L_{11} / \delta_{F}$ or Da, the normalized wave speeds $\bar{S}_{T} / S_{L}$ are close to one another, cf. black circles and triangles.

Fourth, a well-pronounced bending effect, i.e., a decrease in the slope $d \bar{S}_{T} / d U^{\prime}$ with $U^{\prime} / S_{L}$, is shown both by the present DNS data, see black symbols, and by the DNS data by Wenzel and Peters [54,55], see blue pentagons or pluses. Moreover, the values of $\bar{S}_{T} / S_{L}$ obtained at $U^{\prime}>2 S_{L}$ in the two studies are sufficiently close to one another. However, the data by Wenzel and Peters [54,55] indicate leveling off of $\bar{S}_{T}\left(U^{\prime}\right)$ curves, whereas the slope $d \bar{S}_{T} / d U^{\prime}$ is always positive under conditions of the present DNS. This difference could be attributed not only to the use of the $G$ equation in the former case, but also to the fact that a ratio of the axial length of the computational domain to $U^{\prime 3} / \overline{\langle\varepsilon\rangle}$ was about six in the simulations by Wenzel and Peters $[54,55]$, but was as large as about 24 in the present cases characterized by $\kappa_{f} / \kappa_{0}=3$.

The bending effect will further be discussed in Sec. IV B. Before doing so, let us explore dependencies of the computed $\bar{S}_{T}$ on basic mixture and turbulence characteristics, such as $U^{\prime} / S_{L}, L_{11} / \delta_{F}, \mathrm{Da}$, and Ka. For these purposes, the present DNS data were fitted using Eq. (11), where $Y=\left\{\bar{S}_{T} / U^{\prime},\left(\bar{S}_{T}-S_{L}\right) / U^{\prime}, \bar{S}_{T} / S_{L}, \bar{S}_{T} / S_{L}-1\right\}$ and $X=\left\{U^{\prime} / S_{L}, \mathrm{Da}, \mathrm{Ka}, \mathrm{Da} \mathrm{Ka},\left(U^{\prime} / S_{L}\right)\left(L_{11} / \delta_{F}\right)^{q}\right\}$. It is worth remembering that the Schmidt number $v / \mathcal{D}$ was varied in the present study in order to vary a ratio of $L_{11} / \delta_{F}$ by retaining the same $U^{\prime} / S_{L}$ and the same turbulence characteristics $\left(\operatorname{Re}_{t}\right.$, $U^{\prime}, L_{11}$, etc.). Therefore, a function of $U^{\prime} / S_{L}$ and $L_{11} / \delta_{F}$ is not reduced to a function of $\mathrm{Da}$ and $\mathrm{Ka}$.

When using $X=\left(U^{\prime} / S_{L}\right)\left(L_{11} / \delta_{F}\right)^{q}$ to fit the DNS data, the power exponent $q$ was varied from -4 to 4 with a step of 0.01 . Then, parameters $a$ and $b$ were evaluated using a least square fit in logarithmic coordinates for each value of $q$. Finally, the value of $q$ that yielded the highest $R^{2}$ evaluated using Eq. (12) was selected.

Parameters of the five best fits for $\bar{S}_{T} / U^{\prime}$ and $\bar{S}_{T} / S_{L}$ are listed in Table $\mathrm{V}$ and the three best fits characterized by the largest $R^{2}$ are plotted in Fig. 7(b). Results calculated for $\bar{S}_{T}-$ $S_{L}$ are not reported, because subtraction of $S_{L}$ from $\bar{S}_{T}$ reduces $R^{2}$, i.e., impedes fitting, for all $X$. This trend is consistent with

TABLE V. Results of fitting the DNS data on $\bar{S}_{T}: 17$ cases are characterized by $\kappa_{f}=\kappa_{0}=3$ and $L_{11} / L \approx 0.12$, and 22 cases include five more cases characterized by $\kappa_{f}=\kappa_{0}=1$ and $L_{11} / L=$ 0.2 .

\begin{tabular}{|c|c|c|c|c|c|c|c|c|c|}
\hline \multirow[b]{2}{*}{$Y$} & \multirow[b]{2}{*}{$X$} & \multicolumn{4}{|c|}{22 cases } & \multicolumn{4}{|c|}{17 cases } \\
\hline & & $q$ & $\ln a$ & $b$ & $R^{2}$ & $q$ & $\ln a$ & $b$ & $R^{2}$ \\
\hline$\frac{\bar{S}_{T}}{U^{\prime}}$ & $\begin{array}{l}\frac{U^{\prime}}{S_{L}} \\
D a\end{array}$ & & $\begin{array}{r}0.60 \\
-0.10\end{array}$ & $\begin{array}{r}-0.47 \\
0.44\end{array}$ & $\begin{array}{l}0.82 \\
0.88\end{array}$ & & $\begin{array}{r}0.53 \\
-0.09\end{array}$ & $\begin{array}{r}-0.50 \\
0.49\end{array}$ & $\begin{array}{l}0.89 \\
0.94\end{array}$ \\
\hline & $\begin{array}{c}K a \\
\frac{U^{\prime}}{S_{I}}\left(\frac{L_{11}}{\delta_{F}}\right)^{q}\end{array}$ & -0.51 & $\begin{array}{l}0.79 \\
0.23\end{array}$ & $\begin{array}{l}-0.47 \\
-0.49\end{array}$ & $\begin{array}{l}0.93 \\
0.96\end{array}$ & -0.56 & $\begin{array}{l}0.83 \\
0.18\end{array}$ & $\begin{array}{l}-0.50 \\
-0.52\end{array}$ & $\begin{array}{l}0.95 \\
0.98\end{array}$ \\
\hline$\frac{\bar{S}_{T}}{S_{L}}$ & & 0.85 & -0.05 & 0.51 & 0.98 & 1.01 & 0.08 & 0.48 & 0.99 \\
\hline
\end{tabular}

results of fitting various experimental databases on turbulent flame speeds [56].

Table $\mathrm{V}$ shows that two best fits associated with the largest $R^{2}$ yield $\bar{S}_{T} \propto\left(U^{\prime} S_{L}\right)^{0.5}$. Moreover, the best fit yields $\bar{S}_{T} \propto\left(L_{11} / \delta_{F}\right)^{s}$ with $s_{17}=0.49$ if 17 cases characterized by $\kappa_{f} / \kappa_{0}=3$ and $L_{11} / L \approx 0.12$ are analyzed, but $s_{22}=0.43$ if five more cases characterized by $\kappa_{f} / \kappa_{0}=1$ and $L_{11} / L=0.2$ are also analyzed. A similar trend $\left(s_{22}<s_{17}\right)$ is shown by three other fits for $\bar{S}_{T} / U^{\prime}$ as a function of $\mathrm{Da}, \mathrm{Ka}$, or $\left(U^{\prime} / S_{L}\right)\left(L_{11} / \delta_{F}\right)^{q}$.

At first glance, this difference in $s_{17}$ and $s_{22}$ might be attributed to the influence of the width of the computational domain. However, it is also worth noting that any of these fits holds in a bounded range of mixture and turbulence characteristics and should not be extrapolated to $L_{11} / \delta_{F} \rightarrow$ $\infty$. For instance, in the case of infinitely large ratio of $L_{11} / \delta_{F}$, the scaling of $\bar{S}_{T} \propto U^{\prime}$ has been obtained by numerically solving the $G$ equation (5) with $S_{L}=S_{L}^{0}$ [24,54,55]; see the red filled symbols and blue crosses in Fig. 7(a). Accordingly, a decrease in the exponent $s$ with increasing $L_{11} / \delta_{F}$ may be assumed. This is in line with the DNS data, because the five extra cases that affect $s_{22}<s_{17}$ are characterized by the largest $L_{11} / \delta_{F}=12.5$; see Table II. Bearing in mind that (i) neither $L_{11} / \delta_{F}$, see Fig. $4(\mathrm{~b})$, nor $\kappa_{f} / \kappa_{0}$ (or $L_{11} / L$ ), see Table IV, substantially affects $\bar{\delta}_{T}$ under conditions of the present DNS, but (ii) both $L_{11} / \delta_{F}$ and $L_{11} / L$ affect $\bar{S}_{T}, s_{22}<s_{17}$ is associated with the decrease in $s$ with increasing $L_{11} / \delta_{F}$.

The scaling for fully developed mean turbulent wave speed, resulting from the present DNS, i.e., $\bar{S}_{T} \propto\left(U^{\prime} S_{L}\right)^{0.5}\left(L_{11} / \delta_{F}\right)^{q}$ with $0.4<q \leqslant 0.5$, is sufficiently close ${ }^{5}$ to $\bar{S}_{T} \propto U^{\prime} \mathrm{Da}^{1 / 2}$ and is similar (if Sc $=$ const) to scaling $\bar{S}_{T} \propto S_{L} \operatorname{Re}^{1 / 2}$ yielded by several models of premixed turbulent combustion [57-59]. A similar scaling was documented in various experiments, e.g., $\bar{S}_{T} \propto S_{L} \operatorname{Re}^{1 / 2}$ [60], $\bar{S}_{T} \propto U^{\prime} \mathrm{Da}^{1 / 2}$ [61], $\bar{S}_{T} \propto U^{\prime} \mathrm{Da}^{0.44}$, see data by Kido et al. [62] fitted by Lipatnikov and Chomiak [56], $\bar{S}_{T} \propto U^{\prime 0.63} S_{L}^{0.37}\left(L_{11} / \delta_{F}\right)^{0.58}$ [63], or $\bar{S}_{T} \propto$ $U^{\prime 0.67} S_{L}^{0.33}\left(L_{11} / \delta_{F}\right)^{0.41}$, see data by Kobayashi et al. $[64,65]$ fitted by Lipatnikov and Chomiak [56].

Nevertheless, there are experimental databases on turbulent flame speeds that support other power-law fits. In particular, as shown by Lipatnikov and Chomiak [56], the three most extensive experimental databases obtained from expanding statistically spherical premixed flames [66-68] are reasonably well fitted with $\bar{S}_{T} \propto U^{\prime} \mathrm{Ka}^{-1 / 3}$ or $\bar{S}_{T} \propto U^{\prime} \mathrm{Da}^{1 / 4}$ and, therefore, indicate a less pronounced dependence of $\bar{S}_{T} / U^{\prime}$ on Ka or Da when compared to the present DNS. Differences between these measured data and the present DNS results could be attributed to a number of factors, such as (i) thermal expansion effects, reviewed elsewhere $[69,70]$, (ii) differences between experimental and numerical flame configurations, (iii) differences between methods adopted to evaluate $\bar{S}_{T}$ in the experiments and simulations [71], etc.

\section{B. Bending effect}

While the bending effect is well documented in experiments with premixed turbulent flames, as reviewed

\footnotetext{
${ }^{5}$ Data that support this scaling are reported in the appendix.
} 
elsewhere [56,72], explanation and modeling of this effect is still the grand challenge to the combustion community.

Because the sole difference between the previous [24] and present DNS consists in substituting the $G$ equation (5) with Eq. (3), the obtained difference between the linear and bent $\bar{S}_{T}\left(U^{\prime}\right)$ curves cannot be attributed to the exploited method of turbulence generation, numerical resolution or scheme, insufficiently large width of the computational domain, etc. For instance, cases $10-37$ and $10-40-L_{11} / L$ are characterized by significantly different (by a factor of two) ratios of $L_{11} / L$, see Table I, with all other things being approximately equal, but, in spite of the difference in $L_{11} / L$, the values of $\overline{\delta_{T}} / L_{11}$ or $\bar{S}_{T} / U^{\prime}$, computed in the two cases, are approximately equal, see the last two columns in Tables II and III. Therefore, the difference between the $\bar{S}_{T}\left(U^{\prime}\right)$ curves [or filled red and open black symbols in Fig. 7(a)] is straightforwardly associated with a nonzero local thickness of the reaction wave, which was addressed in the present DNS, but was neglected in the previous simulations, with all other things being equal. Accordingly, explanation of the bending effect shown in Fig. 7(a) should be based on physical mechanisms that stem from the nonzero $\delta_{F}$.

In the rest of the present subsection, a role played by three such physical mechanisms is considered. It is worth stressing that we will solely discuss physical mechanisms that might eventually explain the difference between the linear and bent $\bar{S}_{T}\left(U^{\prime}\right)$ curves computed in the previous [24] and present DNS. For the sake of brevity, this difference will be called the bending effect in the rest of the present subsection.

\section{Local reaction extinction?}

In the combustion literature, deviation of dependencies of flame speed on rms turbulent velocity from a straight line is most often attributed to local decrease in burning rate and local combustion quenching due to high stretch rates created by small-scale turbulent eddies [73-75].

At first glance, such a physical mechanism is not relevant to the conditions of the present DNS. For instance, in the case of (i) single-step chemistry, (ii) Le $=1$, and (iii) adiabatic burning, high stretch rates cannot cause local combustion extinction, i.e., holes in a flame surface. Indeed, due to continuity of a $c$ field and continuous, single-valued (in the considered case) dependence of the rate $W$ on $c$, any curve that connects a point in products $(c=1)$ and a point in fresh reactants $(c=0)$ inevitably crosses a reaction zone $c_{1} \leqslant c \leqslant c_{2}$, where the reaction rate is high [13]. Moreover, within the framework of the asymptotic $(\mathrm{Ze} \rightarrow \infty)$ theory of weakly stretched, adiabatic laminar premixed flames [76,77], consumption velocity $u_{c}$, i.e., the reaction rate $W$ integrated along the local normal to the reaction wave, is not affected by flame stretch rate if Le $=1$ and chemistry is single step. However, local variations in $u_{c}$ can occur in the case of a nonzero thickness of the reaction zone, i.e., at large but finite Ze [78]. Therefore, a role played eventually by such a physical mechanism under conditions of the present DNS should be addressed.

A straightforward way to assessing a role played by this physical mechanism consists in processing DNS data on the local $u_{c}$. However, in intense turbulence, topology of reaction zone is so complicated, see Fig. 3, that application of this
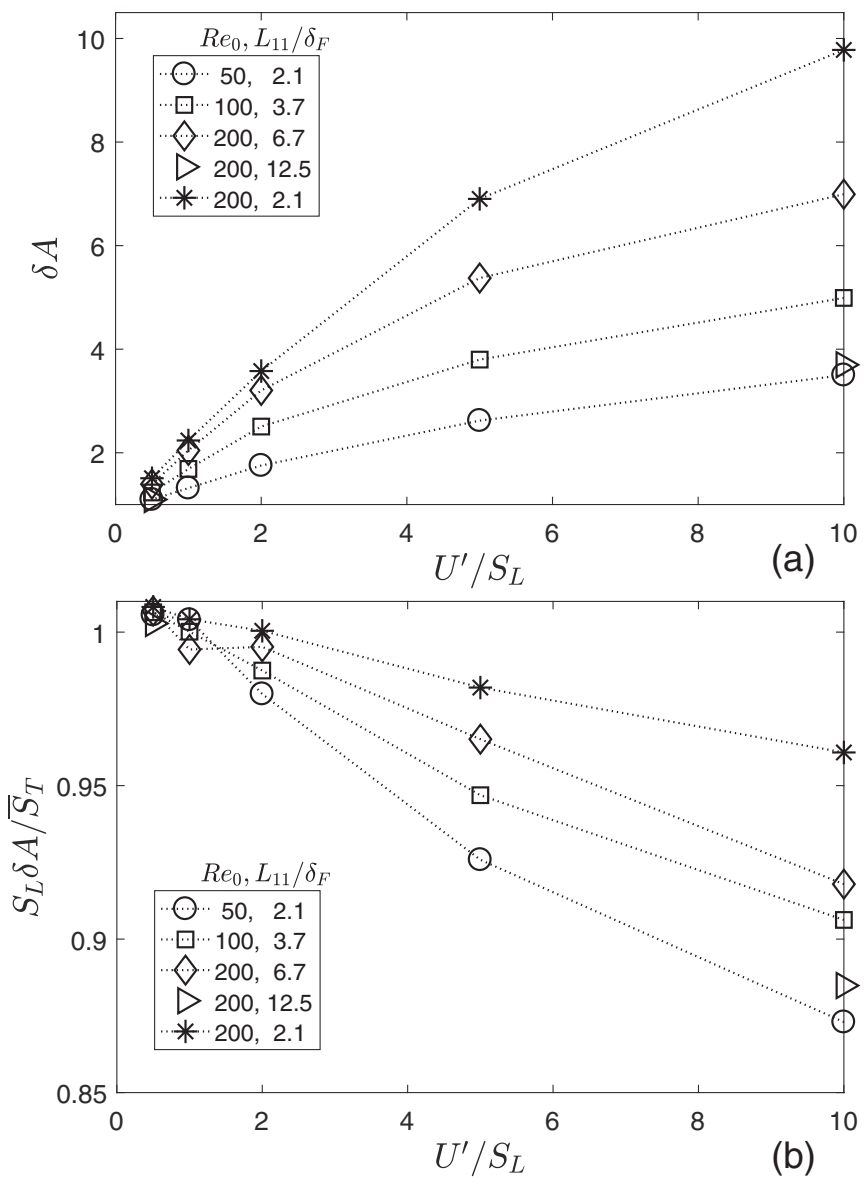

FIG. 8. Dependencies of (a) the relative area increase $\delta A$ given by Eq. (16) and (b) a ratio of $S_{L} \delta A / \bar{S}_{T}$ on $U^{\prime} / S_{L}$. Symbol legends are explained in caption to Fig. 4.

method is difficult. Accordingly, here, the issue is addressed using an indirect method.

To characterize an increase $\delta A=A_{T} / A_{L}$ in the area of the surface of a reaction zone in a turbulent flow when compared to the laminar wave, the following ratio is invoked:

$$
\delta A=\frac{\int_{0}^{L_{x}} \overline{\langle|\nabla c|\rangle_{f}}[\bar{c}(x)] d x}{\int_{x\left(c_{1}\right)}^{x\left(c_{2}\right)}|\nabla c|_{L} d x},
$$

where the symbol $\overline{\langle\cdot\rangle_{f}}(\bar{c})$ designates value of flame surface density $|\nabla c|$ [2] conditioned to the reaction zone, as defined by Eq. (9), and the denominator is evaluated by integrating the laminar profile $c_{L}(x)$ over the reaction zone, i.e., at $c_{1} \leqslant c \leqslant c_{2}$.

Figure 8(a) shows that the bending of the $\delta A\left(U^{\prime} / S_{L}\right)$ curves is well pronounced. Moreover, these curves look similar to the $\bar{S}_{T} / S_{L}\left(U^{\prime} / S_{L}\right)$ curves plotted in Fig. 7(a), thus implying that the bending of the latter curves is mainly controlled by the bending of the former curves. Indeed, a ratio of $\left(S_{L} \delta A\right) / \bar{U}_{T}$, shown in Fig. 8(b), is close to unity, thus further supporting statistically negligible variations in $u_{c}$ under conditions of the present DNS. Furthermore, contrary to eventual increase in $\left(S_{L} \delta A\right) / \bar{U}_{T}$ by $U^{\prime} / S_{L}$ due to eventual reduction in $\bar{u}_{c}$ by turbulent stretching, the ratio of $\left(S_{L} \delta A\right) / \bar{U}_{T}$ is slightly decreased when $U^{\prime} / S_{L}$ is increased. This effect could be 
attributed to (i) an increase in the burning rate integrated over mixing zones $\left(c<c_{1}\right)$, as such zones are expanded by small-scale turbulent eddies [57], or (ii) an increase in the local burning rate in the vicinity of cusps [79]. In any case, the increase in $\left(S_{L} \delta A\right) / \bar{U}_{T}$ by $U^{\prime} / S_{L}$ does not contribute to the bending effect, but weakly resists it.

Thus, the discussed bending of $\bar{S}_{T}\left(U^{\prime}\right)$ curves appears to be controlled by reduction of the rate of an increase in the area of reaction-zone surface with increasing $U^{\prime} / S_{L}$. This conclusion is consistent with earlier DNS studies by Wenzel and Peters [54,55] and Nivarti and Cant [21]. In the next two subsubsections, two physical mechanisms apparently relevant to such a behavior of the reaction-zone surface area are addressed.

\section{Restriction of wave propagation by high strain rates?}

By considering premixed turbulent combustion at high Karlovitz numbers, Kuznetsov and Sabelnikov [13] have hypothesized that influence of turbulent strain rates on local burning can manifest itself not only in the local flame extinction but also in pushing the flame out spatial regions characterized by high strain rates. Such a phenomenon may bound the flame expansion, impede growing its surface area, and therefore reduce $S_{T}$. Law and Sung [80] also emphasized that a flame can survive by moving out spatial regions characterized by high strain rates.

In order to assess a role played eventually by such a physical mechanism, we computed PDF $P\left(S^{2}\right)$ by analyzing the entire flow field and the counterpart PDF

$$
\begin{aligned}
\bar{P}_{f}\left(S^{2}\right)= & \int_{0}^{1} \int_{c_{1}}^{c_{2}} \bar{P}\left(S^{2}, c, \bar{c}\right) d c d \bar{c} / \\
& \int_{-\infty}^{+\infty} \int_{0}^{1} \int_{c_{1}}^{c_{2}} \bar{P}\left(S^{2}, c, \bar{c}\right) d c d \bar{c} d S^{2}
\end{aligned}
$$

conditioned to the reaction zone. Here, $S^{2}=S_{i j} S_{i j}$ is the total strain. Results reported in Fig. 9 show that, at high values of $S^{2}$, the conditioned PDF is larger than the unconditioned one;

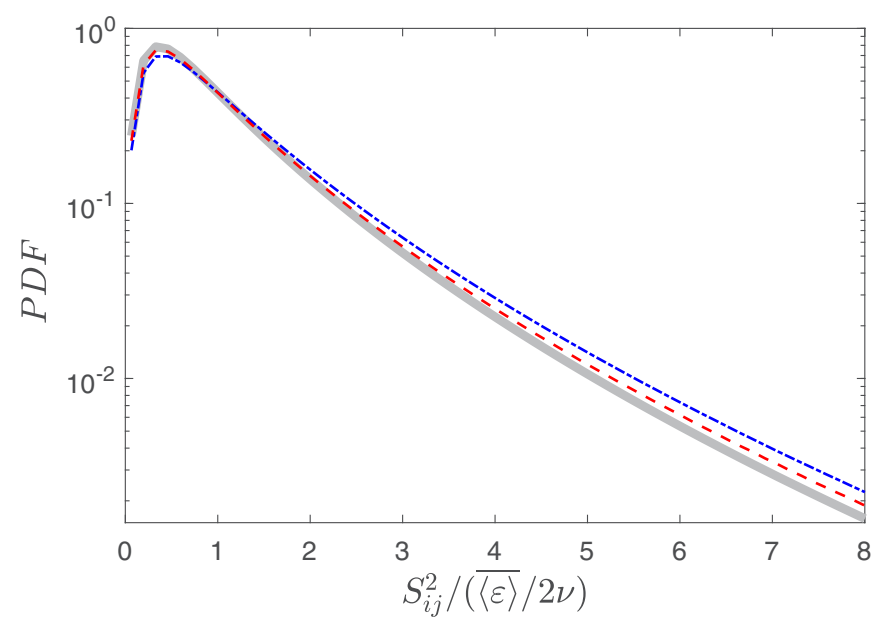

FIG. 9. PDFs for the total strain $S^{2}$ normalized using $\overline{\langle\varepsilon\rangle} /(2 v)$. Gray thick solid line shows the unconditioned $P\left(S^{2}\right)$. Red dashed and blue dot-dashed lines show the conditioned PDFs $\bar{P}_{f}\left(S^{2}\right)$ obtained in cases 05-67 and 10-67, respectively; see Table II. cf. the curve shown by the broken line with the curve shown by the thick solid gray line. The difference is more pronounced at $U^{\prime} / S_{L}=10$, see curve shown in blue dot-dashed line, when compared to $U^{\prime} / S_{L}=0.5$, see curve shown in red dashed line. Accordingly, Fig. 9 does not indicate that reaction zones bypass regions characterized by high strain rates.

Moreover, the sign of the displacement speed [1,2]

$$
S_{d}=\frac{\mathcal{D} \nabla^{2} c+W}{|\nabla c|}
$$

conveys some information about eventual restriction of propagation of reaction zones. Indeed, a locally negative value of conditioned $\left\langle S_{d} \mid c_{1} \leqslant c \leqslant c_{2}\right\rangle$ is associated with reduced capability of the reaction zone to enter this spatial region. Accordingly, we extracted $4 \mathrm{D}$ PDFs $\bar{P}\left(S_{d}, S^{2}, c, \bar{c}\right)$ and $\bar{P}\left(S_{d}, k_{m}, c, \bar{c}\right)$ from the DNS data and computed probability

$$
\begin{aligned}
\overline{\mathrm{P}}_{f, S_{d}<0}\left(S^{2}\right)= & \int_{-\infty}^{0} \int_{0}^{1} \int_{c_{1}}^{c_{2}} \bar{P}\left(S^{2}, S_{d}, c, \bar{c}\right) d c d \bar{c} d S_{d} / \\
& \int_{-\infty}^{+\infty} \int_{0}^{1} \int_{c_{1}}^{c_{2}} \bar{P}\left(S^{2}, S_{d}, c, \bar{c}\right) d c d \bar{c} d S_{d}
\end{aligned}
$$

or

$$
\begin{aligned}
\overline{\mathrm{P}}_{f, S_{d}<0}\left(k_{m}\right)= & \int_{-\infty}^{0} \int_{0}^{1} \int_{c_{1}}^{c_{2}} \bar{P}\left(k_{m}, S_{d}, c, \bar{c}\right) d c d \bar{c} d S_{d} / \\
& \int_{-\infty}^{+\infty} \int_{0}^{1} \int_{c_{1}}^{c_{2}} \bar{P}\left(k_{m}, S_{d}, c, \bar{c}\right) d c d \bar{c} d S_{d}
\end{aligned}
$$

of finding negative $S_{d}$, conditioned to the local total strain $S^{2}$ or curvature $k_{m}=\nabla \cdot \boldsymbol{n}$ of reaction-zone surface, respectively. Here, $\boldsymbol{n}=-\nabla c /|\nabla c|$ is the unit normal vector. A method of extracting 4D PDFs $\bar{P}\left(q_{1}, q_{2}, c, \bar{c}\right)$ was basically similar to the sampling method of extracting 3D PDFs $\bar{P}(q, c, \bar{c})$, described in Sec. III. However, the former PDFs were obtained by processing 20 stored statistically independent fields of $c\left(\boldsymbol{x}, t^{m}\right)$, $\boldsymbol{u}\left(\boldsymbol{x}, t^{m}\right)$, and $p\left(\boldsymbol{x}, t^{m}\right)$. Here, $m=1,2, \ldots, 20, t^{1}>t_{*}$, and $t^{m}>t^{m-1}+\tau_{t}^{0}$.

Figure 10(a) shows that the probability of finding negative $S_{d}$ depends weakly on total strain even if $S^{2}$ is so large that the probability of finding such a value of $S^{2}$ is very low, cf. Figs. 9 and 10(a). This result implies that turbulent strain rates affect propagation of the reaction zone with respect to the local flow weakly from the statistical viewpoint.

All in all, Figs. 9 and 10(a) do not evidence that reaction zone is pushed out regions characterized by high strain rates. On the contrary, Fig. 10(b) implies an important role played by the local curvature of the reaction zone, as discussed in the next section.

\section{Smoothing mechanism}

Due to molecular transport processes, a wave of a nonzerothickness $\delta_{F}$ smooths out local wrinkles whose length scale is sufficiently small. For instance, applications of fractal analysis to instantaneous flame surfaces have indicated that the lowest scale of the surface wrinkles is controlled by $\delta_{F}[3,81]$.

To illustrate such a smoothing mechanism by considering a simple case following Zeldovich et al. [82], let us rewrite 

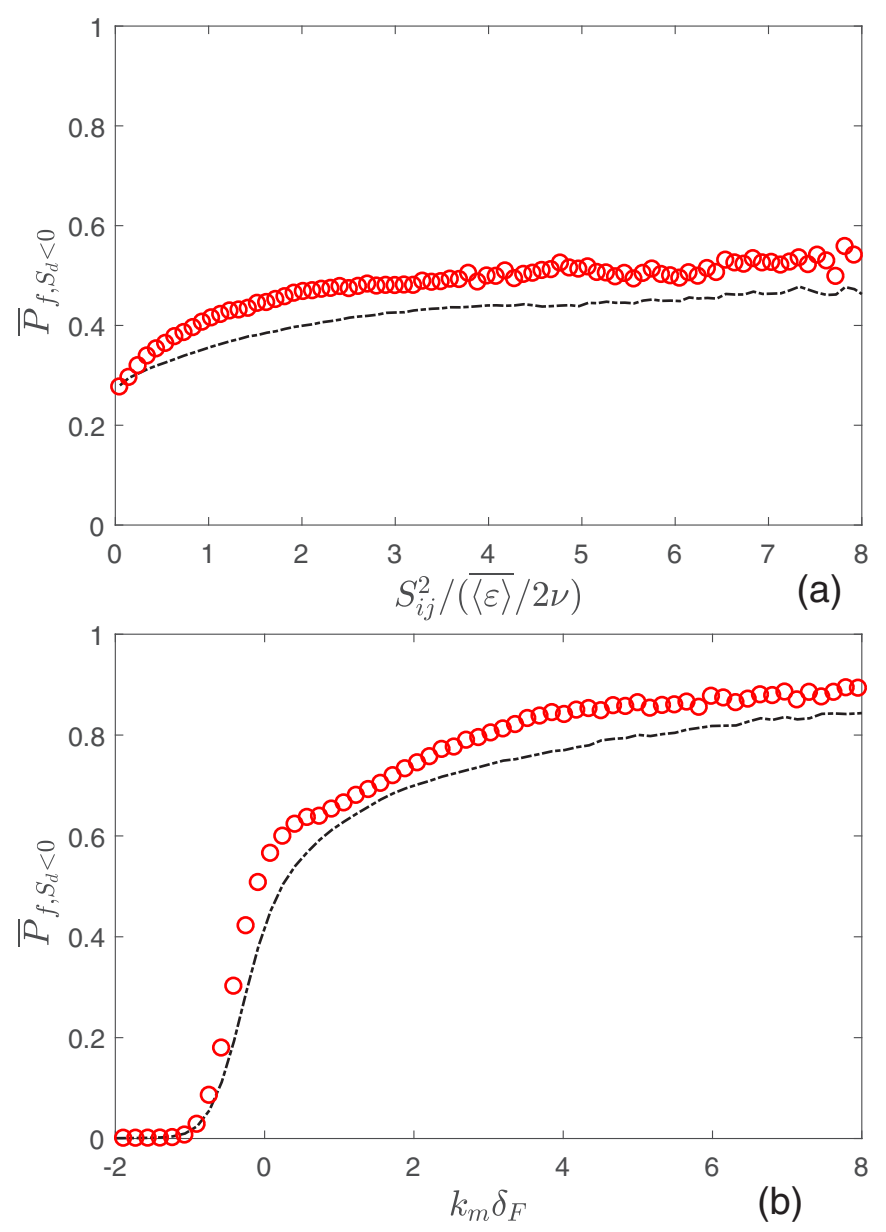

FIG. 10. Probabilities of negative displacement speeds conditioned to the reaction zone and either (a) total strain $S^{2}$ normalized using $\overline{\langle\varepsilon\rangle} /(2 v)$ or (b) curvature $k_{m}$ normalized using the laminar wave thickness $\delta_{F}$. Black dashed line and red circles show results obtained in cases $10-67-4 \delta$ and $10-67-4 \delta-Z e$, see Table III, which are associated with the most pronounced bending effect.

Eq. (3) in the spherical coordinate system

$$
\frac{\partial c}{\partial t}+\frac{1}{r^{2}} \frac{\partial}{\partial r}\left(r^{2} u\right)-\frac{2 \mathcal{D}}{r} \frac{\partial c}{\partial r}=\mathcal{D} \frac{\partial^{2} c}{\partial r^{2}}+W .
$$

For an expanding reaction wave, the last term on the left-hand side reduces the wave speed when compared to the counterpart planar wave. If the local radius $r_{c}$ of the wave curvature is on the order of the Kolmogorov length scale $\eta<\delta_{F}$, the magnitude $\mathcal{D} / r_{c}$ of the negative speed resulting from the considered term is on the order of the Kolmogorov velocity [if $\mathrm{Sc}=O(1)$ or even larger if $\mathrm{Sc}$ is low] and can be much larger than $S_{L}$ if $\mathrm{Ka} \gg 1$. This curvature-induced speed is negative (positive) for a local wrinkle with curvature center in products (reactants) and, therefore, tends to smooth out the wrinkle. This physical mechanism is controlled by the molecular diffusion and acts even if the local consumption velocity $u_{c}$ does not depend on $r_{c}$. Moreover, while a turbulent eddy whose length scale is significantly smaller than $\delta_{F}$ perturbs the local wave surface during a short lifetime of the eddy, the considered mechanism can smooth out the perturbation even after the disappearance of the eddy until $\mathcal{D} / r_{c}=O\left(S_{L}\right)$ and $r_{c}=O\left(\delta_{F}\right)$. Accordingly, if $\eta \ll \delta_{F}$, the small-scale range of the entire turbulence spectrum may be inefficient in wrinkling the wave surface and this inefficient range expands to smaller length scales when $U^{\prime}$ is increased. This physical mechanism acts to impede increasing wave surface area and $\bar{S}_{T}$ with increasing $U^{\prime}$.

Indeed, Fig. 10(b) shows that, in cases 10-67-4 $\delta$ and $10-67-4 \delta-\mathrm{Ze}$, associated with the most pronounced bending effect, see black triangles in Fig. 7(a), the probability of finding negative $S_{d}$ is strongly increased by $k_{m}$ if $\left|k_{m} \delta_{F}\right|<1$. The probability is larger than $70 \%$ if $k_{m} \delta_{F}>2$. Accordingly, if the local curvature of a reaction zone is positive and sufficiently high $\left(k_{m}>\delta_{F}^{-1}\right)$, the zone statistically tends (i) to move to products, i.e., to the curvature center, and therefore (ii) to smooth out the local wrinkle of the zone surface. If the local curvature of a reaction zone is negative and sufficiently high $\left(k_{m}<-\delta_{F}^{-1}\right)$, the zone moves to unburned gas [the probability of finding positive $S_{d}$ is almost equal to unity; see Fig. 10(b)], i.e., to the curvature center, and therefore smooths out the local wrinkle of the zone surface again.

Moreover, Fig. 11(a) shows that the probability

$$
\overline{\mathrm{P}}_{f}\left(\left|k_{m}\right|>k_{m}^{*}\right)=1-\int_{-k_{m}^{*}}^{k_{m}^{*}} \int_{c_{1}}^{c_{2}} \int_{0}^{1} \bar{P}\left(k_{m}, c, \bar{c}\right) d c d \bar{c} d k_{m}
$$

of finding highly wrinkled reaction zones characterized by $\left|k_{m}\right|>k_{m}^{*}=b \eta^{-1}$ at $c_{1}<c<c_{2}$, where $b$ is a positive number of unity order, is significantly increased when the laminar wave thickness $\delta_{F}$ is decreased, cf. results computed in cases $100-21-4 \delta, 10-21$, or by solving the $G$ equation (5), but depends weakly on Ze, cf. cases $10-21-4 \delta$ and $10-21-4 \delta-Z e$.

Figure 11(b) indicates that the probability of finding highly wrinkled reaction zones characterized by $\left|k_{m}\right|>k_{m}^{*}=b \delta_{F}^{-1}$ is weakly affected by variations in $\mathrm{Re}_{0}, \mathrm{Ka}, \delta_{F} / \eta$, and Ze, provided that $U^{\prime} / S_{L}$ and $L_{11} / \delta_{F}$ are kept constant. In the cases addressed in Fig. 11(b), variations in the thickness $\delta_{F}=\mathcal{D} / S_{L}$ are controlled by variations in the diffusivity $\mathcal{D}$. Consequently, significant (weak) dependence of the discussed probability on $\delta_{F}$ (Ze and, hence, the reaction zone thickness) implies that the molecular transport impedes wrinkling reaction-zone surface by small-scale eddies in intense turbulence.

Thus, the above analysis of the present DNS data indicates that the bending of $\bar{S}_{T} / U^{\prime}$ curves, computed in the case of a nonzero local thickness of reaction wave, is controlled by the bending of the mean area of reaction-zone surface as a function of $U^{\prime} / S_{L}$; see Fig. 8(a). The latter bending is controlled by the following physical mechanism: When a reaction front has a negligible thickness, see the red symbols in Fig. 7(a), turbulent eddies of various scales can wrinkle the front surface, increase its area, and hence increase $\bar{S}_{T}$, e.g., the red solid curve in Fig. 11(a). However, if the local thickness $\delta_{F}$ of a reaction wave is comparable with or larger than the Kolmogorov length scale $\eta$, the local molecular transport efficiently smooths out smallscale wrinkles of the wave surface, cf. other results shown in Fig. 11(a) with results shown by the magenta solid curve. Therefore, the local molecular transport impedes increasing the surface area due to the highest local stretch rates created by the smallest turbulent eddies. Consequently, $\bar{S}_{T}$ is reduced 

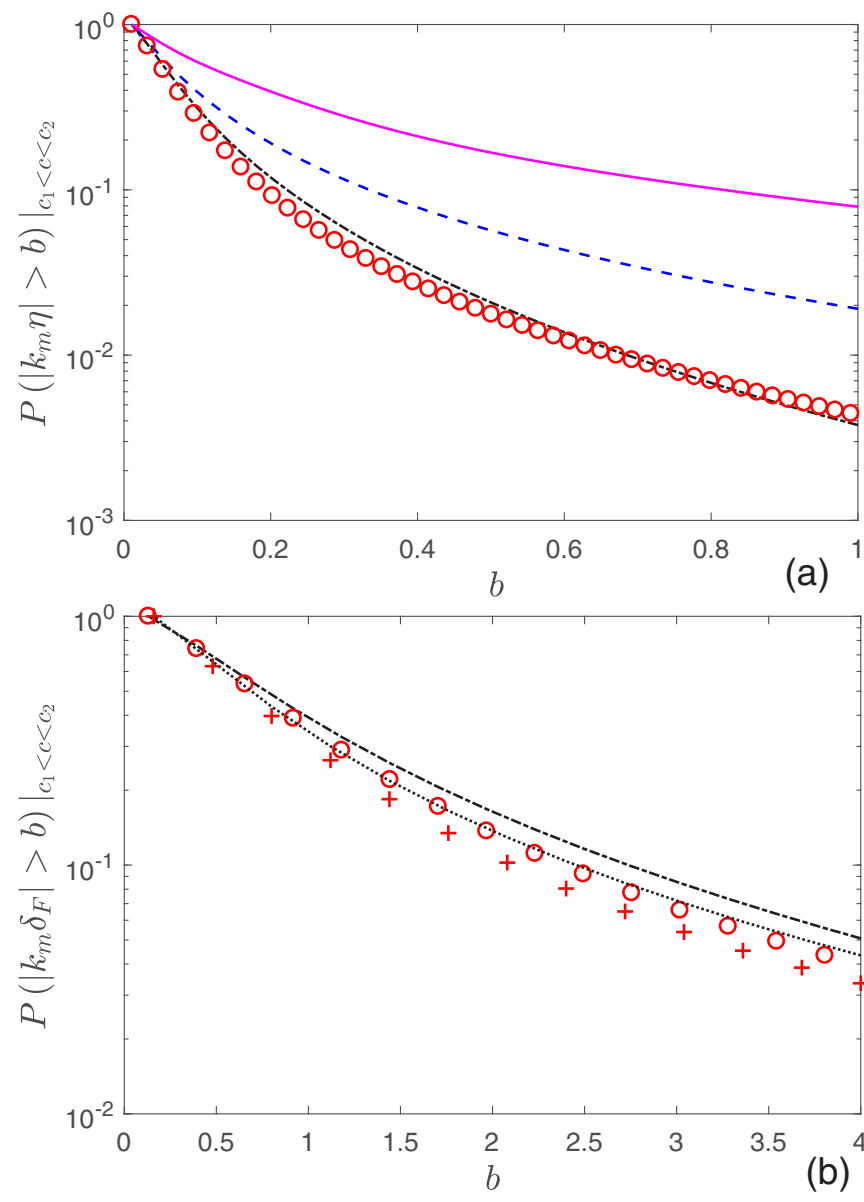

FIG. 11. Cumulative probability that the normalized absolute value of local curvature of reaction zone is larger than a positive number $b$. (a) Curvature is normalized using Kolmogorov length scale. All results were obtained in cases associated with the same turbulence $\left(\operatorname{Re}_{0}=200\right.$ and $\left.U^{\prime} / S_{L}=10\right)$, but different $L_{11} / \delta_{F}=2.1$ (short-dashed line, case 10-21-4 $\delta$ or red circles, case 10-21-4 $\delta$-Ze), 6.7 (long-dashed line, case 10-67), and $\infty$ (solid line, $G$ equation). (b) Curvature is normalized using $\delta_{F}=\mathcal{D} / S_{L}$. All results were obtained in cases associated with the same $L_{11} / \delta_{F}$, but different Ze and different turbulent flow fields. Dashed line, circles, pluses, and dotted line show results obtained in cases $10-21-4 \delta, 10-21-4 \delta-\mathrm{Ze}, 10-21^{*}-\mathrm{Ze}$, and $10-21^{*}$, respectively.

when compared to the case of an infinitely thin reaction wave, cf. black and red symbols in Fig. 7(a).

The present simulations show that this physical mechanism plays an important role even in the case of a reaction wave that does not affect the flow. Therefore, this physical mechanism differs fundamentally from the well-known reduction of the influence of the small-scale turbulent stretching on a premixed flame, which (reduction) stems from destruction of the smallest turbulent eddies due to combustion-induced dilatation and an increase in the viscosity $[48,49]$.

Under conditions of the present DNS, the highlighted physical mechanism appears to play a role in all cases characterized by a nonzero $\delta_{F}$, because $\delta_{F}>\eta$ in all these cases; see Tables II and III. Under conditions of a typical experiment with premixed turbulent flames, such a mechanism can reduce turbulent flame speed $S_{T}$ if the turbulence length scale $L_{11}$ is sufficiently small or the rms velocity $U^{\prime}$ is sufficiently large. For instance, if $L_{11} \gg \delta_{F}$ is kept constant and $U^{\prime}$ is increased starting from a sufficiently low value such that $\eta \gg \delta_{F}$, then the discussed mechanism does not seem to play a role at low $U^{\prime}$, but reduces $S_{T}$ when $U^{\prime}$ reaches a critical value such that $\eta \approx \delta_{F}$.

\section{CONCLUSIONS}

A DNS study of propagation of a thin reaction wave in forced, constant-density, statistically stationary, homogeneous, isotropic turbulence was performed by solving NavierStokes and reaction-diffusion equations under a wide range of conditions that covers various (from 0.5 to 10.0 ) ratios of the rms turbulent velocity $U^{\prime}$ to the laminar wave speed $S_{L}$, various (from 2.1 to 12.5 ) ratios of the integral length scale $L_{11}$ of the turbulence to the laminar wave thickness $\delta_{F}$, and two Zeldovich numbers, Ze $=6.0$ and 17.1. Accordingly, the Damköhler Da and Karlovitz Ka numbers were varied from 0.2 to 25.1 and from 0.4 to 36.2 , respectively. Computed data were compared with previous DNS results [24] obtained by tracking an infinitely thin $\left(\mathrm{Ze}=\mathrm{Da}=L_{11} / \delta_{F}=\infty\right.$, whereas $\left.\mathrm{Ka}=0\right)$ self-propagating front in statistically the same turbulence.

On the one hand, comparison of the present and previous DNS data does not show a substantial effect of the local wave thickness $\delta_{F}$ on the mean wave thickness or the mean turbulent flux $\overline{u^{\prime} c^{\prime}}$ of a reaction progress variable $c$. In particular, the following effects simulated in the present work are similar to effects revealed in the previous DNS study [24] of self-propagation of an infinitely thin front in the statistically the same turbulence. First, the normalized fully developed mean wave thickness $\bar{\delta}_{T} / L_{11}$ is increased by $U^{\prime} / S_{L}$, i.e., $\bar{\delta}_{T} \propto L_{11}\left(U^{\prime} / S_{L}\right)^{b}$, where $b$ is close to 0.4 ; see Fig. 4 and Table IV. Second, the fully developed flux $\overline{u^{\prime} c^{\prime}}$ is affected by the chemical reaction. In particular, diffusivity $\overline{D_{T}^{*}}$ associated with the flux and averaged over the fully developed wave brush is decreased when a ratio of $U^{\prime} / S_{L}$ is decreased; see Fig. 6(a). On the contrary, a ratio of $\bar{D}_{T}^{*} /\left(U^{\prime} \bar{\delta}_{T}\right)$ depends weakly on $U^{\prime} / S_{L}$; see Fig. 6(b).

On the other hand, the mean wave speed is significantly reduced when $\delta_{F} / L_{11}$ is increased. The mean wave speed computed in the present study scales as follows: $\bar{S}_{T} \propto$ $\left(U^{\prime} S_{L}\right)^{0.5}\left(L_{11} / \delta_{F}\right)^{q}$, with $q$ being close to 0.5 . In particular, the obtained DNS data are well fitted with $\bar{S}_{T} \propto U^{\prime} \mathrm{Da}^{1 / 2}$, thus highlighting the chemical time scale $\tau_{c}=\delta_{F} / S_{L}$ to be the mixture characteristic that controls the fully developed mean turbulent wave speed. Moreover, the following results obtained in the present work stem from a nonzero thickness $\delta_{F}$ of the reaction wave.

First, the computed $\bar{S}_{T} / S_{L}\left(U^{\prime} / S_{L}\right)$ curves show bending; see Fig. 7(a). The bending effect is increased by $\delta_{F} / L_{11}$, but vanishes in the case of an infinitely thin front.

Second, under conditions of the present study, the bending effect is controlled by a decrease in the rate of an increase $\delta A$ in the reaction-zone surface area with increasing $U^{\prime} / S_{L}$; see Fig. 8. In its turn, the bending of the $\delta A\left(U^{\prime} / S_{L}\right)$ curves stems from inefficiency of small-scale turbulent eddies in wrinkling the reaction-zone surface, because small-scale wrinkles are 
smoothed out by molecular transport within the local reaction wave; see Figs. 10(b) and 11. Such a smoothing effect was not observed in the DNS of self-propagation of an infinitely thin front at a constant speed $S_{L}$ [24].

Finally, the mean wave thickness and speed are weakly affected by an increase in the Zeldovich number Ze from 6.0 to 17.1; see the last two columns in Tables II and III.

It is worth stressing that simulations discussed in the present paper are the second step in an ongoing DNS series aiming at improving understanding of the governing physical mechanisms of premixed turbulent combustion by investigating a set of basic problems starting from the simplest ones addressed in previous work [24] and the present work. Subsequently, we plan (i) to complicate the problem step by step by allowing for preferential diffusion and Lewis number effects, density variations, and finally complex combustion chemistry and (ii) to reveal a role played by each of these effects by straightforwardly comparing data obtained in two subsequent sets of the DNS series.

\section{ACKNOWLEDGMENTS}

R.Y. gratefully acknowledges the financial support of the Swedish Research Council (VR E0542501). A.N.L. gratefully acknowledges the financial support of the Chalmers Combustion Engine Research Center (CERC) and Chalmers Areas of Advance Transport and Energy. The authors are very grateful to Prof. Vladimir Sabelnikov for fruitful discussion. The computations were performed using the computer facilities provided by the Centre for Scientific and Technical Computing at Lund University (Lunarc). Moreover, the computations were also performed on resources provided by the Swedish National Infrastructure for Computing (SNIC) at Beskow-PDC Center and Triolith-NSC Center.

\section{APPENDIX: BENDING EFFECT AT LARGER VALUES OF $U^{\prime} / S_{L}$}

After submission of the first version of the present paper, new simulations were run in cases characterized by larger values of $U^{\prime} / S_{L}$; see Table VI. Those cases were based on a turbulence field, which was slightly modified when compared to the field addressed in the third line of Table I. The point is that the computed turbulent wave brush was wide at high $U^{\prime} / S_{L}$; cf. the column $\bar{\delta}_{T} / L_{11}$ in Tables VI and II. Accordingly, in order for the computational domain to include the entire wave brush, the axial length $L_{x}$ of the domain and the number $N_{x}$ of grid cells in the $x$ direction were doubled; i.e., a

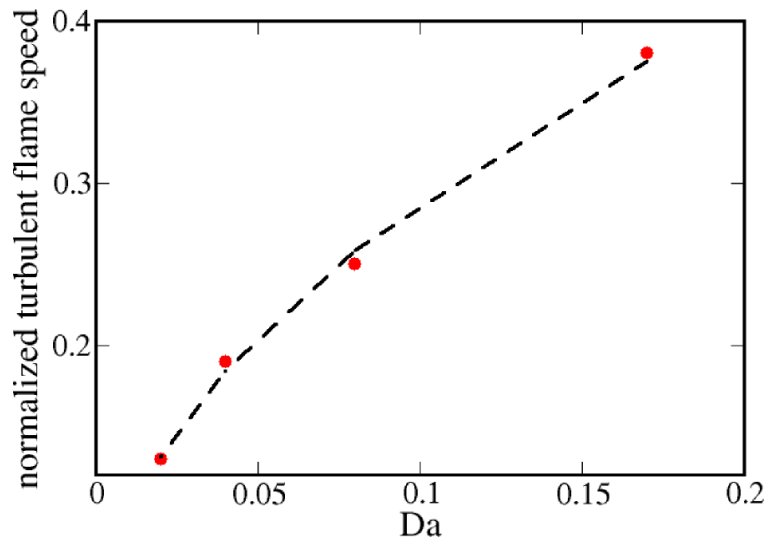

FIG. 12. Normalized turbulent flame speed $\bar{S}_{T} / U^{\prime}$ vs Damköhler number Da. Circles show DNS data. Line shows the following fitting $\bar{S}_{T} / U^{\prime}=0.9 \mathrm{Da}^{1 / 2}$.

rectangular box of $L_{x}=8 L_{y}=8 L_{z}$ was resolved using a uniform staggered Cartesian grid of $2056 \times 256^{2}$ cells. This modification did not change the turbulence statistics when compared to the statistics reported in the third line of Table I.

The DNS data reported in the next-to-last column in Table VI clearly show the bending effect, i.e., a decrease in $\bar{S}_{T} / U^{\prime}$ with increasing $U^{\prime} / S_{L}$. Moreover, these data agree well with a scaling of $\bar{S}_{T} \propto U^{\prime} \mathrm{Da}^{1 / 2}$, which fits to the DNS data computed at $U^{\prime} / S_{L} \leqslant 10$ and is discussed in the main body of the present paper. First, this scaling can be rewritten in the form $\bar{S}_{T} \propto S_{L} \mathrm{Pe}^{1 / 2}$, where $\mathrm{Pe}=u^{\prime} L_{11} /\left(S_{L} \delta_{F}\right)$ is the Péclet number, which is equal to 69 in the four new cases, as well as in the basic case 10-67 addressed in the 15th row in Table II. Accordingly, a ratio of $\bar{S}_{T} / S_{L}$ is expected to be the same in the five (the basic and four new) cases. Indeed, the last column in Table VI shows that this ratio retains approximately the same value (7.6 in the basic case) when increasing $U^{\prime} / S_{L}$ and $\mathrm{Ka}$, decreasing $L_{11} / \delta_{F}$ and Da, but retaining the same value of Pe.

Second, the obtained equality of the normalized speeds $\bar{S}_{T} / S_{L}$ computed at the same Pe is consistent with approximately equal values of $\bar{S}_{T} / U^{\prime}$ or $\bar{S}_{T} / S_{L}$ simulated in cases $05-21$ and $05-21-\delta$ or $10-21$ and $10-21-\delta$. Indeed, two cases $05-21$ and $05-21-\delta$ are characterized by the same Pe $=1.05$ and two cases $10-21$ and $10-21-\delta$ are also characterized by the same $\mathrm{Pe}=21$.

Third, Fig. 12 indicates that the scaling of $\bar{S}_{T} \propto S_{L} \mathrm{Pe}^{1 / 2} \propto$ $U^{\prime} \mathrm{Da}^{1 / 2}$ fits the new DNS data well. These and other new DNS data will be discussed in detail in subsequent papers.

TABLE VI. Four new DNS cases.

\begin{tabular}{|c|c|c|c|c|c|c|c|c|c|c|c|c|}
\hline Case & $\operatorname{Re}_{0}$ & $\kappa_{f} / \kappa_{0}$ & $L_{11} / \mathrm{L}_{y}$ & $\mathrm{Ze}$ & $U^{\prime} / S_{L}$ & $L_{11} / \delta_{F}$ & $\mathrm{Da}$ & $\mathrm{Ka}$ & $\delta_{F} / \eta$ & $\bar{\delta}_{T} / L_{11}$ & $\bar{S}_{T} / U^{\prime}$ & $\bar{S}_{T} / S_{L}$ \\
\hline $20-35$ & 200 & 3 & 0.11 & 6.0 & 20.0 & 3.46 & 0.17 & 43.3 & 7.44 & 6.19 & 0.38 & 7.6 \\
\hline $30-24$ & 200 & 3 & 0.11 & 6.0 & 30.0 & 2.35 & 0.08 & 97.4 & 11.2 & 7.16 & 0.25 & 7.5 \\
\hline $40-18$ & 200 & 3 & 0.11 & 6.0 & 40.0 & 1.76 & 0.04 & 173. & 14.9 & 8.67 & 0.19 & 7.6 \\
\hline $60-12$ & 200 & 3 & 0.11 & 6.0 & 60.0 & 1.16 & 0.02 & 390. & 22.3 & 11.7 & 0.13 & 7.8 \\
\hline
\end{tabular}


[1] N. Peters, Turbulent Combustion (Cambridge University Press, Cambridge, UK, 2000).

[2] T. Poinsot and D. Veynante, Theoretical and Numerical Combustion (Edwards, Philadelphia, PA, 2005).

[3] A. Lipatnikov, Fundamentals of Premixed Turbulent Combustion (CRC Press, Boca Raton, FL, 2012)

[4] H. Kolla, E. R. Hawkes, A. R. Kerstein, N. Swaminathan, and J. H. Chen, J. Fluid Mech. 754, 456 (2014).

[5] B. Yenerdag, N. Fukushima, M. Shimura, M. Tanahashi, and T. Miyauchi, Proc. Combust. Inst. 35, 1277 (2015).

[6] H. Carlsson, R. Yu, and X.-S. Bai, Proc. Combust. Inst. 35, 1425 (2015).

[7] S. Chaudhuri, Phys. Rev. E 91, 021001(R) (2015).

[8] D. Cecere, E. Giacomazzi, N. M. Arcidiacono, and F. R. Picchia, Combust. Flame 165, 384 (2016).

[9] A. J. Aspden, M. S. Day, and J. B. Bell, Combust. Flame 166, 266 (2016).

[10] S. Lapointe and G. Blanquart, Combust. Flame 167, 294 (2016).

[11] M. Day, S. Tachibana, J. Bell, M. Lijewski, V. Beckner, and R. K. Cheng, Combust. Flame 162, 2148 (2015).

[12] H. Wang, E. R. Hawkes, B. Zhou, J. H. Chen, Z. Li, and M. Aldén, Proc. Combust. Inst. 36, 2045 (2017).

[13] V. R. Kuznetsov and V. A. Sabelnikov, Turbulence and Combustion (Hemisphere, New York, 1990).

[14] V. Bychkov, Phys. Rev. E 68, 066304 (2003).

[15] F. Creta, R. Lamioni, P. E. Lapenna, and G. Troiani, Phys. Rev. E 94, 053102 (2016).

[16] N. Fogla, F. Creta, and M. Matalon, Combust. Flame 175, 155 (2017).

[17] C. Dopazo, L. Cifuentes, J. Martin, and C. Jimenez, Combust. Flame 162, 1729 (2015).

[18] A. N. Lipatnikov, J. Chomiak, V. A. Sabelnikov, S. Nishiki, and T. Hasegawa, Proc. Combust. Inst. 35, 1401 (2015).

[19] A. Y. Poludnenko, Phys. Fluids 27, 014106 (2015).

[20] C. A. Z. Towery, A. Y. Poludnenko, J. Urzay, J. O’Brien, M. Ihme, and P. E. Hamlington, Phys. Rev. E 93, 053115 (2016).

[21] G. Nivarti and S. Cant, Proc. Combust. Inst. 36, 1903 (2017).

[22] S. H. Kim, Proc. Combust. Inst. 36, 2017 (2017).

[23] R. Yu, A. N. Lipatnikov, and X. S. Bai, Phys. Fluids 26, 085104 (2014).

[24] R. Yu, X.-S. Bai, and A. N. Lipatnikov, J. Fluid Mech. 772, 127 (2015).

[25] R. C. Aldredge, Combust. Flame 106, 29 (1996).

[26] L. Kagan and G. Sivashinky, Combust. Flame 120, 222 (2000).

[27] L. Kagan, P. D. Ronney, and G. Sivashinky, Combust. Theory Modelling 6, 479 (2002).

[28] L. Kagan and G. Sivashinky, Combust. Flame 142, 235 (2005).

[29] A. R. Kerstein, W. T. Ashurst, and F. A. Williams, Phys. Rev. A 37, 2728 (1988).

[30] R. Yu, J. Yu, and X.-S. Bai, J. Comput. Phys. 231, 5504 (2012).

[31] F. Zhang, R. Yu, and X.-S. Bai, Int. J. Hydrog. Energy 37, 17285 (2012).

[32] R. Yu and X.-S. Bai, Combust. Flame 160, 1706 (2013).

[33] J. F. Yu, R. Yu, X. Q. Fan, M. Christensen, A. A. Konnov, and X.-S. Bai, Combust. Flame 160, 1276 (2013).

[34] H. Carlsson, R. Yu, and X.-S. Bai, Int. J. Hydrog. Energy 39, 20216 (2014).

[35] G.-S. Jiang and C.-W. Shu, J. Comput. Phys. 126, 202 (1996).

[36] R. Yu and X.-S. Bai, Int. J. Num. Methods Fluids 71, 13 (2013).

[37] R. Yu and X.-S. Bai, J. Comput. Phys. 256, 234 (2014).
[38] A. G. Lamorgese, D. A. Caughey, and S. B. Pope, Phys. Fluids 17, 015106 (2005).

[39] V. Eswaran and S. B. Pope, Comput. Fluids 16, 257 (1988).

[40] G. Darrieus, "Propagation d'un front de flamme," presented at LaTechnique Moderne (Paris), and in 1945 at Congres de Mecanique Appliquee (Paris) (unpublished).

[41] L. D. Landau, Acta Physicochim. USSR 19, 77 (1944).

[42] R. Yu, X.-S. Bai, and V. Bychkov, Phys. Rev. E 92, 063028 (2015).

[43] J. F. Driscoll, Prog. Energy Combust. Sci. 34, 91 (2008).

[44] A. W. Skiba, T. M. Wabel, J. E. Temme, and J. F. Driscoll, in 51st AIAA/SAE/ASEE Joint Propulsion Conference, Orlando, FL (AIAA, Reston, VA, 2015), p. 4089.

[45] B. Zhou, C. Brackmann, Z. Li, M. Aldén, and X.-S. Bai, Proc. Combust. Inst. 35, 1409 (2015).

[46] T. M. Wabel, A. W. Skiba, J. E. Temme, and J. F. Driscoll, Proc. Combust. Inst. 36, 1809 (2017).

[47] A. J. Aspden, M. S. Day, and J. B. Bell, J. Fluid Mech. 680, 287 (2011).

[48] T. Poinsot, D. Veynante, and S. Candel, J. Fluid Mech. 228, 561 (1991).

[49] W. L. Roberts, J. F. Driscoll, M. C. Drake, and L. P. Goss, Combust. Flame 94, 58 (1993).

[50] A. M. Klimov, Dokl. Akad. Nauk SSSR 221, 56 (1975).

[51] S. Corrsin, Adv. Geophysics 18, 25 (1975).

[52] A. N. Lipatnikov, Proc. Combust. Inst. 33, 1489 (2011).

[53] T. Elperin, N. Kleeorin, M. Liberman, and I. Rogachevskii, Phys. Rev. E 90, 053001 (2014).

[54] H. Wenzel and N. Peters, Combust. Sci. Technol. 158, 273 (2000).

[55] H. Wenzel and N. Peters, Combust. Sci. Technol. 177, 1095 (2005).

[56] A. N. Lipatnikov and J. Chomiak, Prog. Energy Combust. Sci. 28, 1 (2002).

[57] G. Damköhler, Ber. Bunsen-Ges. Phys. Chem. 46, 601 (1940).

[58] S. Chaudhuri, V. Akkerman, and C. K. Law, Phys. Rev. E 84, 026322 (2011).

[59] S. Chaudhuri, F. Wu, and C. K. Law, Phys. Rev. E 88, 033005 (2013).

[60] K. O. Smith and F. C. Gouldin, AIAA J. 17, 1243 (1979).

[61] C.-C. Liu, S. S. Shy, M.-W. Peng, C.-W. Chiu, and Y.-C. Dong, Combust. Flame 159, 2608 (2012).

[62] H. Kido, T. Kitagawa, K. Nakashima, and K. Kato, Memoirs of the Faculty of Engineering, Kyushu University 49, 229 (1989).

[63] S. Daniele, P. Jansohn, J. Mantzaras, and K. Boulouchos, Proc. Combust. Inst. 33, 2937 (2011).

[64] H. Kobayashi, T. Tamura, K. Maruta, T. Niioka, and F. A. Williams, in Symposium (International) on Combustion, Vol. 26 (Elsevier, Amsterdam, Neth., 1996), pp. 389-396.

[65] H. Kobayashi, Y. Kawabata, and K. Maruta, in Symposium (International) on Combustion, Vol. 27 (Elsevier, Amsterdam, Neth., 1998), pp. 941-948.

[66] V. P. Karpov and E. S. Severin, Combust., Explosion, Shock Waves 16, 41 (1980).

[67] Ö. L. Gülder, in Symposium (International) on Combustion, Vol. 23 (Elsevier, Amsterdam, Neth., 1991), pp. 743-750.

[68] D. Bradley, A. K. C. Lau, and M. Lawes, Philos. Trans. R. Soc. London, Ser. A 338, 359 (1992).

[69] A. N. Lipatnikov and J. Chomiak, Prog. Energy Combust. Sci. 36, 1 (2010). 
[70] V. A. Sabelnikov and A. N. Lipatnikov, Annu. Rev. Fluid Mech. 49, 91 (2017).

[71] S. Verma and A. N. Lipatnikov, Combust. Flame 173, 77 (2016).

[72] A. N. Lipatnikov and J. Chomiak, Prog. Energy Combust. Sci. 31, 1 (2005).

[73] R. G. Abdel-Gayed, K. J. Al-Khishali, and D. Bradley, Proc. R. Soc. London, Ser. A 391, 393 (1984).

[74] K. N. C. Bray and R. S. Cant, Proc. R. Soc. London, Ser. A 434, 217 (1991).

[75] D. Bradley, in Symposium (International) on Combustion, Vol. 24 (Elsevier, Amsterdam, Neth., 1992), pp. 247-262.

[76] P. Clavin, Prog. Energy Combust. Sci. 11, 1 (1985).
[77] M. Matalon, Annu. Rev. Fluid Mech. 39, 163 (2007).

[78] A. M. Klimov, Zhournal Prikladnoi Mekchaniki i Tekhnicheskoi Fiziki 4, 49 (1963).

[79] A. Y. Poludnenko and E. S. Oran, Combust. Flame 158, 301 (2011).

[80] C. K. Law and C. J. Sung, Prog. Energy Combust. Sci. 26, 459 (2000).

[81] Ö. L. Gülder and G. J. Smallwood, Combust. Flame 103, 107 (1995).

[82] I. B. Zeldovich, G. I. Barenblatt, V. B. Librovich, and G. M. Makhviladze, Mathematical Theory of Combustion and Explosions (Consultants Bureau, New York, 1985). 